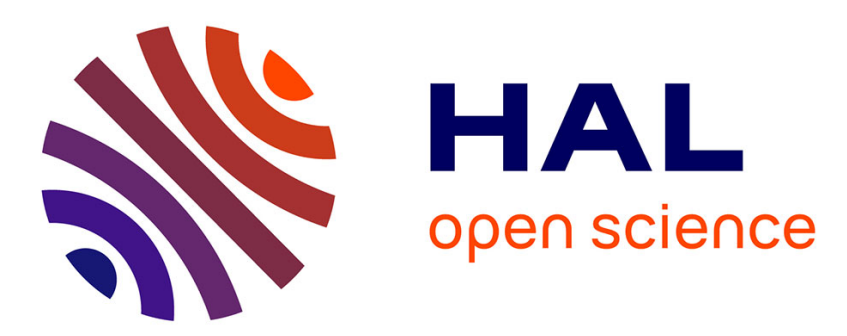

\title{
Vapor-liquid equilibrium of fluids composed by oblate molecules
}

Francisco Gámez, Santiago Lago, Benito Garzón, Patrick Merkling, Carlos Vega

\section{- To cite this version:}

Francisco Gámez, Santiago Lago, Benito Garzón, Patrick Merkling, Carlos Vega. Vapor-liquid equilibrium of fluids composed by oblate molecules. Molecular Physics, 2008, 106 (11), pp.1331-1339. 10.1080/00268970802129826 . hal-00513207

\section{HAL Id: hal-00513207 https://hal.science/hal-00513207}

Submitted on 1 Sep 2010

HAL is a multi-disciplinary open access archive for the deposit and dissemination of scientific research documents, whether they are published or not. The documents may come from teaching and research institutions in France or abroad, or from public or private research centers.
L'archive ouverte pluridisciplinaire HAL, est destinée au dépôt et à la diffusion de documents scientifiques de niveau recherche, publiés ou non, émanant des établissements d'enseignement et de recherche français ou étrangers, des laboratoires publics ou privés. 


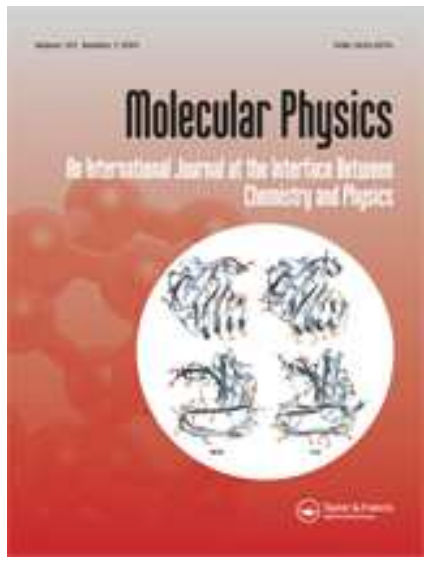

\section{Vapor-liquid equilibrium of fluids composed by oblate molecules}

\begin{tabular}{|c|c|}
\hline Journal: & Molecular Physics \\
\hline Manuscript ID: & TMPH-2008-0047.R2 \\
\hline Manuscript Type: & Full Paper \\
\hline $\begin{array}{l}\text { Date Submitted by the } \\
\text { Author: }\end{array}$ & 14-Apr-2008 \\
\hline Complete List of Authors: & $\begin{array}{l}\text { Gámez, Francisco; Univ.Pablo de Olavide, Physical, Chemical and } \\
\text { Natural Systems } \\
\text { Lago, Santiago; Univ.Pablo de Olavide, Physical, Chemical and } \\
\text { Natural Systems } \\
\text { Garzón, Benito; Universidad San Pablo CEU, Física Aplicada, } \\
\text { Fisicoquímica y Óptica } \\
\text { Merkling, Patrick; Univ.Pablo de Olavide, Physical, Chemical and } \\
\text { Natural Systems } \\
\text { Vega, Carlos; Univ.Complutense, Química Física }\end{array}$ \\
\hline Keywords: & $\begin{array}{l}\text { Vapor-liquid equilibrium, oblate molecules, Boyle temperature, } \\
\text { intermolecular potential. }\end{array}$ \\
\hline
\end{tabular}

\section{S) ScholaroNE"}


7/12/2010 02:49

\title{
Vapour-liquid equilibrium of fluids composed by oblate
}

\section{molecules}

\author{
Francisco Gámez ${ }^{1}$, Santiago Lago ${ }^{1, *}$, Benito Garzón ${ }^{2}$, Patrick Merkling ${ }^{1}$ and Carlos \\ Vega $^{3}$ \\ ${ }^{1}$ Dpto. Sistemas Físicos, Químicos y Naturales. \\ Fac. Ciencias Experimentales, \\ Ctra.Utrera Km.1, \\ Univ. Pablo de Olavide, \\ 41013 Sevilla, Spain \\ ${ }^{2}$ Departamento de Física Aplicada, Fisicoquímica y Óptica, \\ Facultad de Farmacia, \\ Universidad San Pablo CEU, \\ Urb. Monteprincipe Boadilla del Monte 28660, \\ Spain \\ ${ }^{3}$ Dpto. Química Física \\ Fac. CC. Químicas, \\ Univ. Complutense \\ 28040 Madrid, Spain \\ * Author to whom correspondence should be addressed: slagara@upo.es
}




\begin{abstract}
Gibbs ensemble Monte Carlo simulations are performed to obtain the vapourliquid equilibrium of oblate-like fluids interacting through the Kihara intermolecular potential. Our results confirm the validity of a perturbation theory for Kihara fluids, whose accuracy for prolate fluids was tested some years ago. As in the case of hard ellipsoids, the symmetry of the phase diagram of oblate and prolate models is analyzed. An interesting relation of Boyle temperature and critical parameters with molecular volume is found for the considered models. As a particular application, this relation allows the prediction of some thermodynamic properties of a new promising biofuel 2,5dimethylfuran.
\end{abstract}

\title{
Keywords
}

Vapour-liquid equilibrium, oblate molecules, Boyle temperature, intermolecular potential. 


\section{1.- INTRODUCTION}

The liquid state is the most difficult to study among the classical states of matter. Powerful methods to predict thermodynamic and transport properties of liquids have been proposed during the second half of the past century but most of these methods are reliable only for simple fluids [1]. However, most liquids are formed by polyatomic molecules where in addition to translational degrees of freedom, one must also consider rotation and the internal vibrations of the molecule [2]. The inclusion of rotational degrees of freedom, even in rigid models (where vibrations are not considered), requires some additional information about the mass distribution in the molecule or the molecular shape. Classical rotation movement is described using the inertia tensor which can be written as a $3 \times 3$ square matrix. This matrix can be diagonalized and the three eigenvalues define the so-called principal moments of inertia (PMIs) and the eigenvectors define the principal axes of the molecule with origin at the centre of mass of the molecule. Molecules with low symmetry have three different PMIs and are called asymmetric tops [2]. The decay time of several properties of asymmetric tops depends on three diffusion coefficients and five relaxation times [3]. However, experiments can determine only three coefficients or times. In this case, it is very usual to consider that two of the three moments are equal and the third one is a revolution axis for the molecule [3]. This is equivalent to approximate the asymmetric top as a symmetric top [2]. If the principal moment of inertia with respect to the revolution axis is the largest we have an oblate molecule, if it is the smallest, the molecule is called prolate. Whether a molecule is prolate or oblate can be inferred from the experiment. Many real-world molecules can be considered prolate or oblate. Liquids composed by prolate molecules have received a lot of theoretical attention and thermodynamic properties have been 
qualitatively and quantitatively reported for molecules of this geometry. For prolate molecules a number of studies describing liquid crystal transitions [4], vapour-liquid equilibrium [5] and glassy behaviour [6] have been reported. However, relatively little effort has been directed to obtaining properties of oblate-like fluids [7-9]. In some cases, oblate molecules are not described by pure oblate models but rather by related models [10-13]. A new phenomenology appears in oblate molecules, such as discotic liquid crystal phases with short axes parallel to the director, formation of fragile glasses like in o-terphenyl, peculiar surface properties like in asphaltenes [14], .. Oblate-like molecules are candidates for specific molecular architectures, and some of them mainly aromatic carboxylic acids such as 3-hydroxypicolinic or gentisic acid- have absorption, stability and solubility properties suitable for MALDI matrices [14]. In biology, red blood cells are the most common cells with oblate shape. Important gasliquid interchange takes place in the quaternary structure of hemoproteins contained in these cells, where hemo groups embed gas molecules in a reversible way [15]. Even a prolate-oblate transition has been observed in natural systems such as bilayer membranes [16]. Moreover, an oblate molecule, 2,5-dimethylfuran, has been recently proposed as biofuel [17] and we dedicate some of our work to this molecule. From a chemical point of view, it is well-known that the $\pi$-electronic cloud in common oblate systems is often delocalized, yielding chemical reactivity which is so different from that of prolate molecules that they are considered as different functional groups, so called aromatics. Swimming in the very diverse behaviour of oblate-like molecules, our goals in this paper are to answer the following unsolved questions: i) Does molecular shape affect the vapour-liquid equilibrium?; ii) Is it possible to find a symmetry in the phase diagram of oblate and prolate Kihara spherocylinders as found in hard ellipsoids?[18]. In order to solve these questions, this paper is scheduled as follows: section 2 is devoted 
1

2

4

5

6

7

8

9

10

11

12

13

14

15

16

17

18

19

20

21

22

23

24

25

26

27

28

29

30

31

32

33

34

35

36

37

38

39

40

41

42

43

44

45

46

47

48

49

50

51

52

53

54

55

56

57

58

59

60

to present the model and the relevant parameters in prolate and oblate systems. In section 3 we present the simulation procedure and a review of the perturbation theory used in this work. Results related to vapour-liquid equilibrium of some oblate molecules including the new biofuel 2,5-dimethylfuran are shown in section 4. Finally, some remarks and conclusions close the paper in section 5 . 


\section{Molecular model and hard convex systems.}

The Kihara potential describes the interaction between two molecules of any shape as:

$$
u_{i j}^{K}=4 \varepsilon\left[\left(\frac{\sigma}{\rho_{i j}}\right)^{12}-\left(\frac{\sigma}{\rho_{i j}}\right)^{6}\right]
$$

where $\sigma$ and $\varepsilon$ are length and energy parameters and $\rho$ is the shortest distance between molecular hard cores, depending not only on centre-to-centre distance but on mutual molecular orientations. As usual, the core of a spherical molecule is considered to be a dot, for a prolate molecule the core is considered to be a segment and for an oblate molecule the core is an infinitely thin disk, as considered in convex geometry. For more clarity see figure 1. Hard convex bodies and thermodynamic properties have been widely studied and many equations of state have been established [19]. All these equations of state relate thermodynamic quantities with three geometrical descriptors: $(1 / 4 \pi)$ times the mean intrinsic curvature $(\mathcal{R})$, surface $(S)$ and volume $(\mathcal{V})$. Moreover, an analytical equation relates the second virial coefficient of hard convex bodies, $B_{2}$, to the non sphericity parameter $\alpha=\frac{R S}{3 \mathcal{V}}$ in the form:

$$
\frac{B_{2}}{\mathcal{V}}=1+3 \alpha
$$

This equation is a particular case of the general equation: 


$$
B_{2}(T)=\frac{1}{2}\left[V_{c+c}-\int f(\rho) S_{c+\rho+c} d \rho\right]
$$

with $S_{c+\rho+c}=2 S_{c}+8 \pi R_{c} R_{c}+8 \pi\left(R_{c}+R_{c}\right) \rho+4 \pi \rho$

where $f(\rho)=\exp (-\beta u)-1$ is the Mayer function and the geometrical quantities $V_{c+c}$ and $S_{c+\rho+c}$ depends on surface, volume and $(1 / 4 \pi)$ times the mean curvature of the hard convex nucleus. As it has been mentioned, we have considered a hard rod as the core of the prolate model and an infinitely thin disk as the core of the oblate one, whose characteristic parameters are well known to be different. For a given $L^{*}=L / \sigma$ (prolate, see fig.1) or $D^{*}=D / \sigma$ (oblate, see fig.1), the second virial coefficient will be different due to the difference in the geometrical properties (surface, volume and mean radius of curvature of the hard convex core). For prolate molecules the geometrical properties of the core have been given elsewhere $[11,13]$. For oblate molecules they are given by

$$
\begin{aligned}
& R_{c}=\frac{\sigma}{2}\left(1+\frac{\pi D^{*}}{4}\right) \\
& S_{c}=\frac{\pi \sigma^{2}}{2}\left(D^{* 2}+\pi D^{*}+2\right) \\
& V_{c}=\frac{\pi \sigma^{3}}{24}\left(6 D^{* 2}+3 \pi D^{*}+4\right)
\end{aligned}
$$

Hereinafter, we shall use $L^{*}$ to denote the anisotropy of both prolate and oblate molecules (although it should be clear that in the case of oblate molecules $L^{*}$ is just $D^{*}$ ). In figure 2, the Boyle temperature (where the second virial coefficient $\mathrm{B}_{2}$ becomes zero) is plotted as a function of $L^{*}$. It is obvious that for a given value of $L^{*}$ the prolate and oblate Kihara models differ in their Boyle temperatures. However, it is possible to map a prolate model into an oblate model by imposing the condition of equal Boyle temperature, that is: 


$$
T_{B}^{*}\left(L_{1}^{*}\right)=T_{B}^{*}\left(D_{2}^{*}\right)
$$

where $L^{*}$ on the left hand side represents the anisotropy of a prolate model and $D^{*}$ on the right hand side represents the anisotropy of the oblate model. In figure 3, the Boyle temperature of prolate and oblate molecules is plotted as a function of the molecular volume. The very remarkable result is that oblate Kihara molecules and prolate Kihara molecules present the same Boyle temperature when they have the same reduced volume. Thus Eq. (7) is satisfied (approximately) when both the prolate and oblate molecules present the same reduced volume. A new and important question arises now: will the symmetry between prolate and oblate Kihara molecules exhibited at the Boyle temperature be also present in the rest of the phase diagram? This is one of the questions we intend to address here. In the case of hard ellipsoids a symmetry in the phase diagram was found [20] based on the fact that a hard prolate ellipsoid with aspect ratio $\kappa$ has the same volume than an oblate one with aspect ratio $1 / \kappa$. In this work, we shall determine the vapour liquid equilibria for prolate and oblate molecules by computer simulation to explore the existence of symmetry between prolate and oblate models (when described by the Kihara model). It will be shown that when the comparison is made between models having the same molecular volume there is a good mapping between prolate and oblate models 


\section{Computer simulation and perturbation theory.}

\section{3a. Computer simulations.}

In previous works, we have widely established vapour-liquid equilibria of prolate-like Kihara molecules [5], even including multipolar interactions [21,22]. For that purpose, an algorithm able to calculate several millions of $\rho$ per second is used [23]. In this work a well tested algorithm to calculate the minimum distance between disks has been employed [8]. In analogy to the case of prolate molecules, Gibbs ensemble Monte Carlo (GEMC) simulations [24] are developed for systems with three different reduced diameters $L^{*}=L / \sigma$ equal to $0.5233,1$ and 1.2 , in order to compare with prolate models with the same $L^{*}$ value. GEMC were performed with 512 particles, $6000-10000$ cycles for equilibration and $6000-8000$ for averages, truncating the potential when $\rho_{\mathrm{c}}=3 \sigma$ and adding long tail corrections according to expression given by:

$$
\begin{aligned}
& p^{L R}=-\frac{2}{3} n^{2} \pi \int_{\rho_{c}}^{\infty} r_{12}^{3}\left\langle\frac{\partial u\left(r_{12}, \Omega_{1}, \Omega_{2}\right)}{\partial r_{12}}\right\rangle_{g} d r_{12} \\
& U^{L R}=2 n \pi \int_{\rho_{c}}^{\infty} r_{12}^{2}\left\langle u\left(r_{12}, \Omega_{1}, \Omega_{2}\right)\right\rangle_{g} d r_{12}
\end{aligned}
$$

where the subscript $g$ means geometrical average. Furthermore, long range corrections can be obtained analytically [25] by rewriting the previous equations using convex body geometry. 


\section{3b. Perturbation theory.}

A few years ago, we have presented an accurate perturbation theory for Kihara prolate molecules. This theory is a second order perturbation theory based on MoGubbins division [26] of the Kihara potential:

$$
\begin{aligned}
& u_{0}\left(r_{12}, \Omega_{1}, \Omega_{2}\right)= \begin{cases}u^{K}\left(r_{12}, \Omega_{1}, \Omega_{2}\right)+\varepsilon & \rho \leq \sigma \sqrt[6]{2} \\
0 & \rho>\sigma \sqrt[6]{2}\end{cases} \\
& u_{1}\left(r_{12}, \Omega_{1}, \Omega_{2}\right)=\left\{\begin{array}{cc}
-\varepsilon & \rho \leq \sigma \sqrt[6]{2} \\
u^{K}\left(r_{12}, \Omega_{1}, \Omega_{2}\right) & \rho>\sigma \sqrt[6]{2}
\end{array}\right.
\end{aligned}
$$

It allows us to write the Helmholtz free energy up to second order in perturbation expansion as:

$$
\frac{A^{\text {res }}}{N k T}=\frac{A_{0}^{\text {res }}}{N k T}+\frac{A_{1}}{N k T}+\frac{A_{2}}{N k T}
$$

Since the residual free energy is considered to be the one associated to a hard particle with the same radius and diameter, the contribution to Helmholtz free energy is measured by means of a BLIP expansion and integration of the Boublík's equation of state for hard convex body [27], which gives:

$$
\frac{A_{0}^{r e s}}{N k T}=\frac{\eta\left[6 \alpha^{2}-2 \alpha+\eta\left(\frac{3}{2} \alpha(3-5 \alpha)\right)\right]}{(1-\eta)^{2}}+\left(6 \alpha^{2}-5 \alpha-1\right) \ln (1-\eta)
$$

where $\eta=n / V$ is the packing fraction. Perturbation terms can be calculated in different ways: 
1).- The first order perturbation term is calculated by solving the OrnsteinZernike equation with Percus-Yevick (PY), Hypernetted-chain (HNC) or Reference Hypernetted-chain (RHNC) closure with the background correlation function $y\left(r, \Omega_{1}, \Omega_{2}\right)=g\left(r, \Omega_{1}, \Omega_{2}\right) \exp \left[\beta u\left(r, \Omega_{1}, \Omega_{2}\right)\right]$ extracted from Reference Average Mayer function potential (RAM):

$$
\Phi^{R A M}(r)=-\varepsilon T^{*} \ln \int\left\langle u_{0}\left(\mathbf{r}_{\mathbf{1 2}}, \Omega_{1}, \Omega_{2}\right)\right\rangle_{g} d \Omega_{1} d \Omega_{2}
$$

and finally:

$$
\frac{A_{1}}{N k T}=\frac{2 n \pi}{k T} \int_{0}^{\infty} r_{12}^{2}\left\langle u_{1}\left(r_{12}, \Omega_{1}, \Omega_{2}\right) \exp \left(-\beta u_{0}\left(r_{12}, \Omega_{1}, \Omega_{2}\right)\right)\right\rangle_{g} y^{R A M}(r) d \mathbf{r}
$$

In a previous paper, it was shown that similar results for soft repulsive bodies obtained either using the non-sphericity parameter $\alpha$ extracted from the geometrical relationship and the background correlation function from RHNC equation, or using $\alpha$ from virial coefficient (see equation 2) and background correlation function from PY approximation. Due to the faster convergence of the PY formalism, the second choice is used because results obtained with both integral equations are very close.

2).- Two different choices for the second contribution term can be used [28]: local compressibility approximation (LCA) or macroscopic compressibility approximation (MCA): 


$$
\begin{aligned}
& \frac{A_{2}^{L C A}}{N k T}=-\frac{n \pi}{k T}\left(\frac{\partial n}{\partial p}\right) \int_{0}^{\infty} r_{12}^{2}\left\langle u_{1}^{2}\left(r_{12}, \Omega_{1}, \Omega_{2}\right) \exp \left(-\beta u_{0}\left(r_{12}, \Omega_{1}, \Omega_{2}\right)\right)\right\rangle_{g} y^{R A M}(r) d \mathbf{r} \\
& \frac{A_{2}^{M C A}}{N k T}=-\frac{n \pi}{k T}\left(\frac{\partial n}{\partial p}\right)_{0} \frac{\partial}{\partial p}\left(n \int_{0}^{\infty} r_{12}^{2}\left\langle u_{1}^{2}\left(r_{12}, \Omega_{1}, \Omega_{2}\right) \exp \left(-\beta u_{0}\left(r_{12}, \Omega_{1}, \Omega_{2}\right)\right)\right\rangle_{g} y^{R A M}(r) d \mathbf{r}\right)
\end{aligned}
$$

As for the first order term, the convenience of using MCA approximation has been previously tested and will be used in this work.

Once the Helmholtz free energy is determined, one calculates the equation of state and internal energy through:

$$
\begin{aligned}
& Z=1+n^{*}\left(\frac{\partial\left(A^{\text {res }} / N k T\right)}{\partial n^{*}}\right)_{T} \\
& \frac{U^{\text {res }}}{N k}=\left(\frac{\partial\left(A^{\text {res }} / N k T\right)}{\partial(1 / T)}\right)_{n}
\end{aligned}
$$

and the vapour-liquid equilibrium is solved treating vapour phase as a virial expansion up to second order and solving the nonlinear system:

$$
\begin{aligned}
& n_{l} Z_{l}=n_{g}\left(1+n_{g} B_{2}\right) \\
& \frac{A_{l}}{N k T}+Z_{l}+\ln n_{l}=n_{g} B_{2}+\left(1+n_{g} B_{2}\right)+\ln n_{g}
\end{aligned}
$$


As it is well known, analytical perturbation theories cannot predict critical properties, and numerical extrapolation methods are commonly necessary. To overcome this problem, an extrapolation method for the theory already described (IPT) [29] was derived based on deviations between Monte Carlo and theoretical calculations of the first order contribution to Helmholtz free energy, giving very accurate predictions of VLE for prolate Kihara molecules yielding the so-called IPTE.

\section{RESULTS}

In figures 4 and 5, vapour-liquid coexistence of oblate and prolate Kihara molecules respectively for $L^{*}\left(D^{*}\right)=1.0$ are shown. Numerical results for the VLE of simulated models are shown in table 1 for oblate models and in table 2 for prolate models not previously reported. The critical temperature is accurately predicted for both shapes (prolate or oblate) using IPTE as can be observed in table 3. However, liquid density is systematically underestimated in the case of oblate molecules while the agreement for prolate molecules is excellent. Better agreement is obtained in considering coexistence pressures. In panel $b$ of figures 4 and 5, Clausius plots for these models are presented. IPTE data fit very well the simulation results. Furthermore, although densities in the VLE curve are not accurately estimated for oblate models with high anisotropy, critical densities are. As pointed out above, the behaviour of Boyle temperature against molecular volume is universal, but not against characteristic length. In figure 6 , the behaviour of critical parameters against $L^{*}$ is shown, while in figure 7 the plot against molecular volume can be observed. Surprisingly, critical parameters also exhibit universal behaviour against molecular volume, within the simulation error in the case of GEMC results, independently of shape. Table 3 summarizes all the results 
considered here by simulation and perturbation theory. A comparison for both models can be observed plotting critical temperature against Boyle temperature. Thus, knowledge of Boyle temperature could be used as a tool to estimate critical temperature. A plot of critical temperature against Boyle temperature is presented in figure 8 showing the universal character of the relation. This rule can be used when molecular volume is not too high. This plot allows to predict critical properties of important but scarcely studied molecules such as the biofuel 2,5-dimethyl furan [17] whose known thermodynamic properties are practically restricted to its boiling point. In this case, we have checked using a quantum chemical package (gaussian03 at the B3LYP/6-31G**) that the molecule is perfectly planar and the dipole moment is negligible (0.16 Debye). Using van der Waals radii for atoms on this geometry-optimized structure yields $D^{*}=1.6$ and $V_{\mathrm{m}}{ }^{*}=2.83$. From the fit of $T_{\mathrm{c}}{ }^{*}$ vs. $V_{\mathrm{m}}{ }^{*}$ (figure $7 \mathrm{a}$ ), one can interpolate to obtain $T_{\mathrm{c}}{ }^{*}=0.57$. Moreover, using the classical empirical approximation [31] of $\mathrm{T}_{\text {boiling }} / \mathrm{T}_{\text {critical }}=2 / 3$ and the experimental value for boiling temperature of $346 \mathrm{~K}$, one can predict for this biofuel $T_{\mathrm{c}}=550 \mathrm{~K}, T_{\mathrm{B}}=1117 \mathrm{~K}$. Moreover, from the relation between $T_{\mathrm{c}}{ }^{*}$ and $T_{\mathrm{c}}$ an estimated value of $\varepsilon / k=966 \mathrm{~K}$ is found. This value is consistent with the usual values adopted for benzene or cyclohexane in this potential $[9,11]$.

\section{CONCLUSIONS.}

Gibbs ensemble Monte Carlo simulations of oblate and prolate Kihara molecules have been carried out in order to estimate the influence of shape upon vapour-liquid equilibrium. This influence is very subtle and, thus, we have found a similar behaviour of the virial coefficient -i.e. equation of state- for both models when it is plotted against molecular volume. The same behaviour is observed when critical parameters are plotted 
against molecular volume which opens the possibility of predicting molecular properties of poorly studied fluids.

\section{ACKNOWLEDGMENTS}

This work was supported by grants BFM2003-07055, FIS2007-66079-C02-01, and CTQ2007 60910 of the Spanish Ministerio de Educación y Ciencia. 


\section{BIBLIOGRAPHY}

[1] J.P.Hansen and I.R.MacDonald, Theory of Simple Liquids, $3^{\text {rd }}$.ed., Academic Press, 2006

[2] D.A.McQuarrie and J.D.Simon, Physical chemistry. A molecular approach, chapter 18, university Science Books, Sausalito,1997

[3] J.R.Lakowicz, Principles of Fluorescence Spectroscopy, $2^{\text {nd }}$. ed., chapter 12, Kluwer, New York,1999

[4] A. Cuetos, B. Martinez-Haya, L.F. Rull and S. Lago, J.Chem.Phys. 117, 2934-2946, (2002); (E) J.Chem.Phys. 117,11405,(2002).

[5] C.Vega, S.Lago, E.de Miguel and L.F.Rull, J.Phys.Chem.96,7431-7437, (1992).

[6] S.Lago, M.Cortada, M.D.Lobato, F.Gámez, M.Basoalto J.M.Pedrosa and S.Calero, J.Mol.Liq.134,136-141,(2007).

[7] M. Wojcik, K. E. Gubbins, Molec. Phys. 53 (2): 397-420, (1984).

[8] P. Kadlec, J. Janecek, T. Boublik, Molec. Phys. 98 (8): 473-479, (2000).

[9] J. Janecek, T. Boublik, Molec. Phys. 98(11),765-767, (2000).

[10] B. M. Mulder, Molec. Phys. 103 (10): 1411-1424, (2005).

[11] S.Lago and T.Boublík,Coll.Czech.Chem.Commun.,45,3051-3056,(1980).

[12] C.Vega, S.Lago and P.Padilla, J.Phys.Chem.96,1900-1905,(1992).

[13] S.Lago, J.L.López-Martín, B.Garzón y C.Vega, J.Phys.Chem.98,53555361,(1994).

[14] M.D.Lobato, J.M.Pedrosa, A.R.Hortal,B.Martínez-Haya, R.Lebrón-Aguilar and S.Lago, Colloid Surf.A298,72-79,(2007).

[15] A. E. Hoetink, T. J. C. Faes, K. R. Visser, R. M. Heethaar, IEEE Trans. Biomedical Engn. 51 (7): 1251-1261, (2004). 
[16] M. Jaric, U. Seifert, W. Wintz, M. Wortiz. Phys. Rev E., 52, 6623-6634, (1995).

[17] L.D.Schmidt and P.J.Dauenhauer, Nature, 447,914,(2007).

[18] D. Frenkel and B. M. Mulder, Mol. Phys. 55 (5): 1171-1192, (1985).

[19] T.Boublík, Collection Czechoslovak Chem. Commun, vol 51,2301-2432, (1986).

[20] D. Frenkel, B. M. Mulder,J. P. McTague, Phys. Rev. Lett. 52 (4): 287-290, (1984).

[21] B.Garzón,S.Lago, C.Vega and L.F.Rull, J.Chem.Phys.102, 7204-7216, (1995).

[22] B.Garzón, S.Lago, C.Vega, E.de Miguel and L.F.Rull, J.Chem.Phys.101, 4166-4176, (1994).

[23] C.Vega and S.Lago, Comput.Chem.18,55-59, (1994).

[24].-A.Z.Panagiotopoulos, Molec. Phys. 61, 813-826, 1987

[25] C.Vega and D.Frenkel, Molec.Phys., 67,633-650, (1989).

[26] K. C. Mo and K. E. Gubbins. Chem. Phys. Lett. 27: 144-148. (1974).

[27] T.Boublík, Molec. Phys., 42, 209, (1981).

[28] J. A. Barker, D. Henderson. Rev. Mod. Phys. 48 (4): 587-671 1976

[29] C.Vega and S.Lago, Chem.Phys.Lett.185,516-521, (1991).

[30] A. Lotfi, J. Vrabec and J. Fischer, Mol. Phys. 76(6) 1319 (1992).

[31]G.Z.Guldberg, Z.Phys.Chem. 5, 374, (1890). A more accessible reference is R.Digilov and M.Reiner, Eur.J. Phys. 25,15-22 (2004) 


\section{TABLES}

Table 1. GEMC coexistence data for oblate fluids.

\begin{tabular}{|c|c|c|c|}
\hline \multicolumn{4}{|c|}{$D^{*}=0.5233$} \\
\hline$T^{*}$ & $n_{\mathrm{gas}}$ & $n^{*}{ }_{\text {liq }}$ & $p^{*}$ \\
\hline 0.79 & $0.024_{4}$ & $0.23_{2}$ & $0.0143_{5}$ \\
\hline 0.78 & $0.0255_{2}$ & 0.251 & $0.0144_{4}$ \\
\hline 0.775 & $0.0125_{4}$ & $0.243_{4}$ & $0.0083_{4}$ \\
\hline 0.775 & $0.0149_{8}$ & $0.228_{8}$ & $0.0095_{5}$ \\
\hline 0.77 & $0.0155_{5}$ & $0.242_{8}$ & $0.0098_{3}$ \\
\hline 0.75 & $0.0165_{3}$ & $0.26_{1}$ & $0.0099_{3}$ \\
\hline 0.725 & $0.0142_{8}$ & $0.273_{5}$ & $0.0084_{4}$ \\
\hline 0.7 & $0.0110_{6}$ & $0.281_{5}$ & $0.0065_{3}$ \\
\hline 0.675 & $0.0088_{4}$ & $0.290_{4}$ & $0.0051_{2}$ \\
\hline 0.65 & $0.0056_{2}$ & $0.296_{3}$ & $0.002_{2}$ \\
\hline 0.625 & $0.0047_{7}$ & $0.306_{4}$ & $0.002_{2}$ \\
\hline \multicolumn{4}{|c|}{$D^{*}=1$} \\
\hline$T^{*}$ & $n_{\text {gas }}^{*}$ & $n^{*}$ liq & $p^{*}$ \\
\hline 0.68 & $0.0141_{9}$ & $0.11_{1}$ & $0.0070_{4}$ \\
\hline 0.67 & $0.0145_{2}$ & $0.13_{1}$ & $0.0071_{6}$ \\
\hline 0.66 & $0.0104_{5}$ & $0.13_{1}$ & $0.0055_{3}$ \\
\hline 0.66 & $0.0118_{7}$ & 0.1289 & $0.0059_{4}$ \\
\hline 0.65 & $0.0131_{5}$ & $0.140_{5}$ & $0.0062_{5}$ \\
\hline 0.625 & $0.0084_{3}$ & $0.146_{3}$ & $0.0043_{2}$ \\
\hline 0.625 & $0.0087_{3}$ & $0.146_{4}$ & $0.00434_{25}$ \\
\hline 0.6 & $0.0080_{5}$ & $0.1556_{25}$ & $0.0038_{3}$ \\
\hline 0.6 & $0.0065_{2}$ & $0.1565_{25}$ & $0.0032_{2}$ \\
\hline 0.575 & $0.0047_{2}$ & $0.161_{2}$ & $0.00238_{13}$ \\
\hline 0.55 & $0.00583_{9}$ & $0.169_{2}$ & $0.0026_{2}$ \\
\hline 0.525 & $0.0038_{2}$ & $0.173_{1}$ & $0.0017_{1}$ \\
\hline \multicolumn{4}{|c|}{$D^{*}=1.2$} \\
\hline$T^{*}$ & $n^{*}{ }_{\mathrm{gas}}$ & $n^{*}{ }_{\text {liq }}$ & $p^{*}$ \\
\hline 0.425 & $0.00793_{1}$ & $0.1523_{5}$ & $0.0014_{6}$ \\
\hline 0.475 & $0.00872_{3}$ & $0.1495_{6}$ & $0.0016_{7}$ \\
\hline 0.5 & $0.00999_{6}$ & $0.1445_{8}$ & $0.0027_{6}$ \\
\hline 0.525 & $0.0106_{1}$ & $0.1393_{15}$ & $0.0033_{5}$ \\
\hline 0.55 & $0.0107_{1}$ & $0.1359_{14}$ & $0.0037_{4}$ \\
\hline 0.575 & $0.0112_{2}$ & $0.1284_{25}$ & $0.00431_{4}$ \\
\hline 0.6 & $0.01002_{15}$ & $0.120_{2}$ & $0.0043_{4}$ \\
\hline
\end{tabular}


Table 2. GEMC coexistence data for prolate fluids.

a) $L^{*}=0.5233$. Critical parameters are: $T_{\mathrm{c}}^{*}=1.03(2), n_{\mathrm{c}}{ }^{*}=0.18(1)$ and $p_{\mathrm{c}}{ }^{*}=0.054(12)$.

b) $L^{*}=1$. Critical parameters are: $T_{\mathrm{c}}{ }^{*}=0.928(13), n_{\mathrm{c}}{ }^{*}=0.118(4)$ and $p_{\mathrm{c}}{ }^{*}=0.029(5)$.

c) $L^{*}=1.2$. Critical parameters are: $T_{\mathrm{c}}^{*}=0.911(13), n_{\mathrm{c}}{ }^{*}=0.106(5)$ and $p_{\mathrm{c}}{ }^{*}=0.030(14)$.

$L^{*}=0.5233$

\begin{tabular}{cccc}
\hline$T^{*}$ & $n^{*}{ }_{\text {gas }}$ & $n^{*}$ liq & $p^{*}$ \\
\hline 0.95 & $0.0520_{23}$ & $0.31_{2}$ & $0.0328_{15}$ \\
0.925 & $0.043_{2}$ & $0.337_{14}$ & $0.0280_{12}$ \\
0.9 & $0.039_{2}$ & $0.36_{1}$ & $0.025_{1}$ \\
0.875 & $0.0286_{15}$ & $0.364_{13}$ & $0.0194_{9}$ \\
0.85 & $0.024_{2}$ & $0.380_{7}$ & $0.016_{1}$ \\
0.8 & $0.017_{1}$ & $0.399_{6}$ & $0.0112_{7}$ \\
0.775 & $0.012_{1}$ & $0.407_{4}$ & $0.0084_{7}$ \\
0.75 & $0.0094_{6}$ & $0.418_{4}$ & $0.00634_{35}$ \\
\hline
\end{tabular}

$$
L^{*}=1
$$

\begin{tabular}{cccc}
\hline$T^{*}$ & $n^{*}$ gas & $n^{*}{ }_{\text {liq }}$ & $p^{*}$ \\
\hline 0.825 & $0.024_{1}$ & $0.23_{2}$ & $0.0151_{6}$ \\
0.8 & $0.020_{1}$ & $0.246_{9}$ & $0.0123_{6}$ \\
0.775 & $0.0150_{7}$ & $0.261_{7}$ & $0.0095_{4}$ \\
0.75 & $0.0126_{4}$ & $0.272_{7}$ & $0.0079_{2}$ \\
0.725 & $0.010_{1}$ & $0.281_{6}$ & $0.0063_{5}$ \\
0.7 & $0.0083_{4}$ & $0.289_{3}$ & $0.0051_{2}$ \\
0.675 & $0.0058_{3}$ & $0.297_{4}$ & $0.0036_{2}$ \\
0.65 & $0.0051_{3}$ & $0.306_{3}$ & $0.0030_{2}$ \\
0.625 & $0.00343_{23}$ & $0.312_{4}$ & $0.0020_{1}$ \\
0.6 & $0.0028_{5}$ & $0.3194_{25}$ & $0.0016_{2}$ \\
\hline
\end{tabular}

\begin{tabular}{cccc}
\multicolumn{4}{c}{$L^{*}=1.2$} \\
\hline$T^{*}$ & $n^{*}{ }_{\text {gas }}$ & $n^{*}$ liq $_{1}$ & $p^{*}$ \\
\hline 0.7 & $0.0095_{17}$ & $0.249_{3}$ & $0.0055_{8}$ \\
0.675 & $0.0074_{4}$ & $0.259_{4}$ & $0.0043_{2}$ \\
0.65 & $0.0055_{4}$ & $0.265_{4}$ & $0.0032_{2}$ \\
0.625 & $0.00435_{24}$ & $0.275_{3}$ & $0.0025_{1}$ \\
0.6 & $0.0029_{2}$ & $0.2794_{35}$ & $0.0016_{1}$ \\
0.575 & $0.00195_{3}$ & $0.287_{3}$ & $0.00107_{2}$ \\
0.55 & $0.00160_{5}$ & $0.291_{3}$ & $0.00084_{3}$ \\
0.5 & $0.0014_{1}$ & $0.3050_{25}$ & $0.00066_{6}$ \\
0.475 & $0.00079_{4}$ & $0.3100_{25}$ & $0.00037_{2}$ \\
0.7 & $0.010_{2}$ & $0.2496_{34}$ & $0.0055_{8}$ \\
\hline
\end{tabular}


Table 3. Critical properties of oblate fluids.

\begin{tabular}{|c|c|c|c|c|c|c|c|}
\hline$D^{*}$ & $T_{\mathrm{c}} *($ IPTE $)$ & $T_{\mathrm{c}} *(\mathrm{GEMC})$ & $n_{\mathrm{c}}{ }^{*}($ IPTE $)$ & $n_{\mathrm{c}}^{*}($ GEMC) & $p_{\mathrm{c}}^{*}($ IPTE $)$ & $p_{\mathrm{c}}^{*}(\mathrm{GEMC})$ & $Z_{c}$ \\
\hline 0 & & $1.310^{\mathrm{a}}$ & & $0.314^{\mathrm{a}}$ & & $0.126^{\mathrm{a}}$ & \\
\hline 0.3 & $1.06_{2}$ & & $0.169_{9}$ & & $0.061_{15}$ & & 0.342 \\
\hline 0.42 & $0.974_{12}$ & & $0.143_{5}$ & & $0.052_{3}$ & & 0.373 \\
\hline 0.5233 & $0.923_{7}$ & $0.89_{3}$ & $0.121_{4}$ & $0.122_{13}$ & $0.041_{4}$ & $0.030_{14}$ & 0.367 \\
\hline 0.6 & $0.88_{2}$ & & $0.110_{4}$ & & $0.036_{4}$ & & 0.351 \\
\hline 0.8 & $0.808_{7}$ & & $0.082_{2}$ & & $0.029_{6}$ & & 0.438 \\
\hline 0.82 & $0.800_{6}$ & & $0.080_{2}$ & & $0.027_{6}$ & & 0.422 \\
\hline 1 & $0.745_{11}$ & $0.74_{3}$ & $0.064_{1}$ & $0.066_{8}$ & $0.023_{4}$ & $0.010_{5}$ & 0.482 \\
\hline 1.08 & $0.70_{2}$ & & $0.061_{2}$ & & $0.018_{4}$ & & 0.418 \\
\hline 1.2 & $0.68_{2}$ & $0.77_{6}$ & $0.054_{5}$ & $0.05_{2}$ & $0.019_{5}$ & $0.009_{3}$ & 0.518 \\
\hline 1.345 & $0.653_{15}$ & & $0.047_{1}$ & & $0.014_{5}$ & & 0.456 \\
\hline
\end{tabular}

${ }^{a}$ data taken from ref. 30 


\section{FIGURE CAPTIONS}

Figure 1. Molecular cores of a) spherical molecules; b) linear (prolate) molecules and c) oblate molecules.

Figure 2. Boyle temperature against elongation (prolate model) or diameter (oblate model).

Figure 3. Boyle temperature against molecular volume of prolate and oblate models obtained by IPTE.

Figure 4. VLE properties of prolate Kihara fluids with $L^{*}=1.0$ calculated by IPTE (solid symbols) and GEMC (solid symbols). a) Coexistence densities. b) vapour pressures.

Figure 5. VLE properties of oblate Kihara fluids with $D^{*}=1.0$ calculated. a) Coexistence densities. b) vapour pressures. Symbols as in figure 4

Figure 6. Critical properties of several prolate and oblate fluids with different sizes. a) Temperatures. b) Densities. c) Pressures. Solid symbols as in figure 4.

Figure 7. Dependence of critical parameters on molecular volume for oblate and prolate Kihara fluids.

Figure 8. Critical versus Boyle temperatures for oblate and prolate Kihara models as obtained by IPTE. 


\section{FIGURES}

\section{Figure 1.}

a)

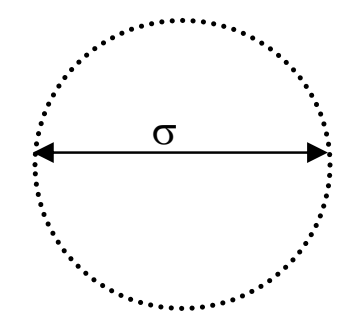

b)

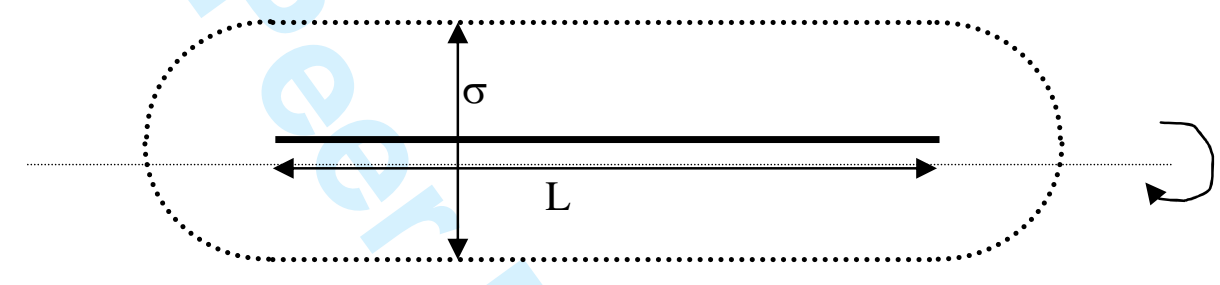

c)

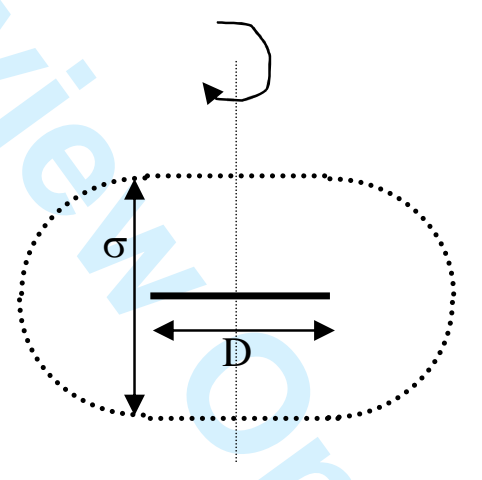

Figure 2. 
Molecular Physics 
Figure 3.

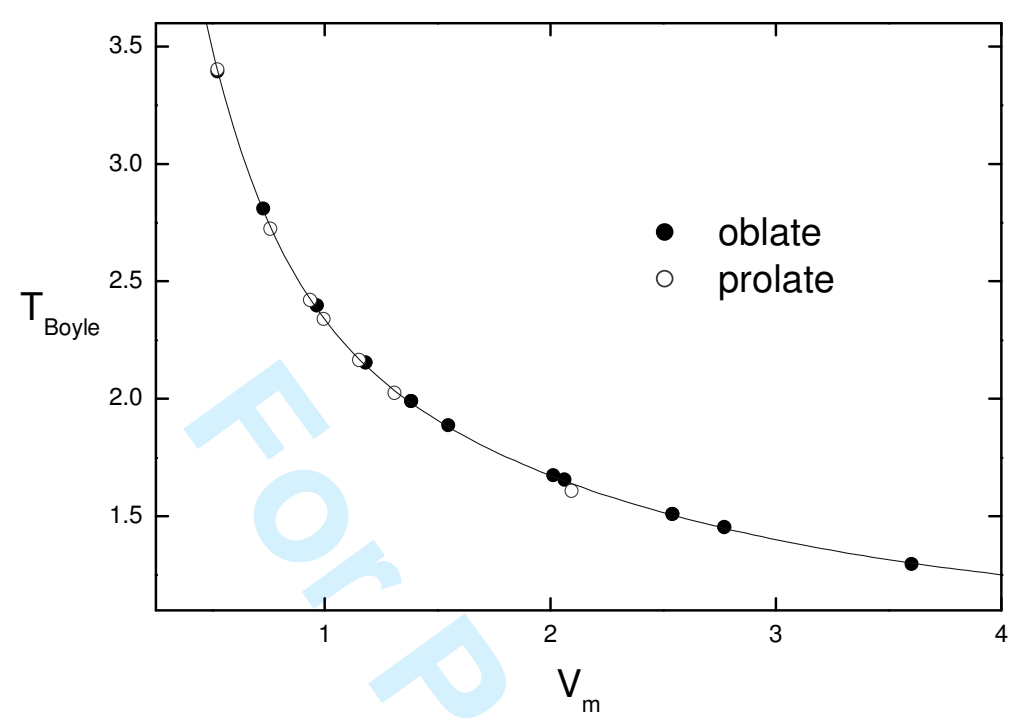

Figure 4.

(a)

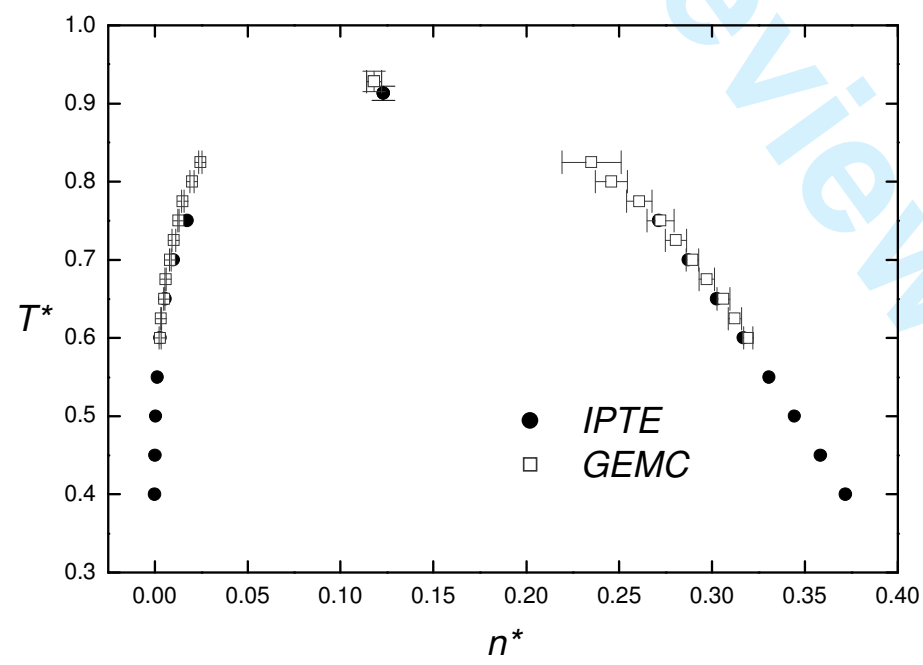

URL: http://mc.manuscriptcentral.com/tandf/tmph 


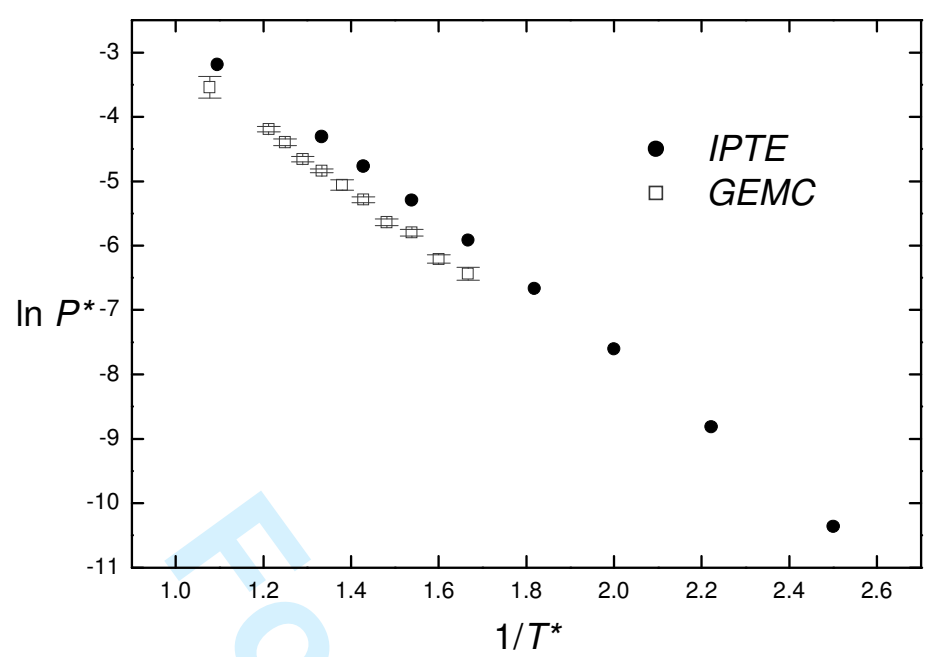

Figure 5.

(a)

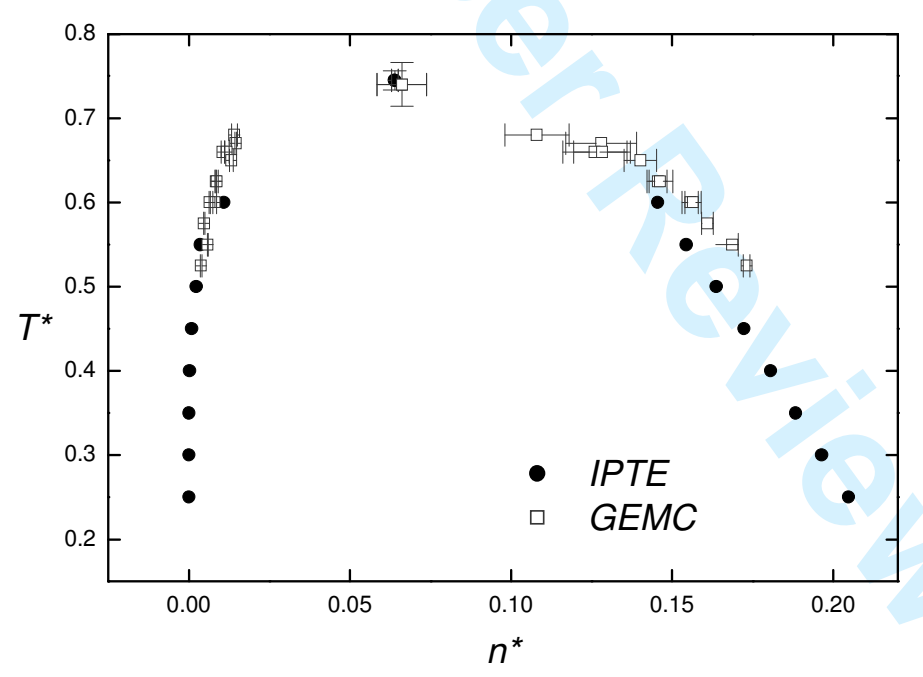

(b) 


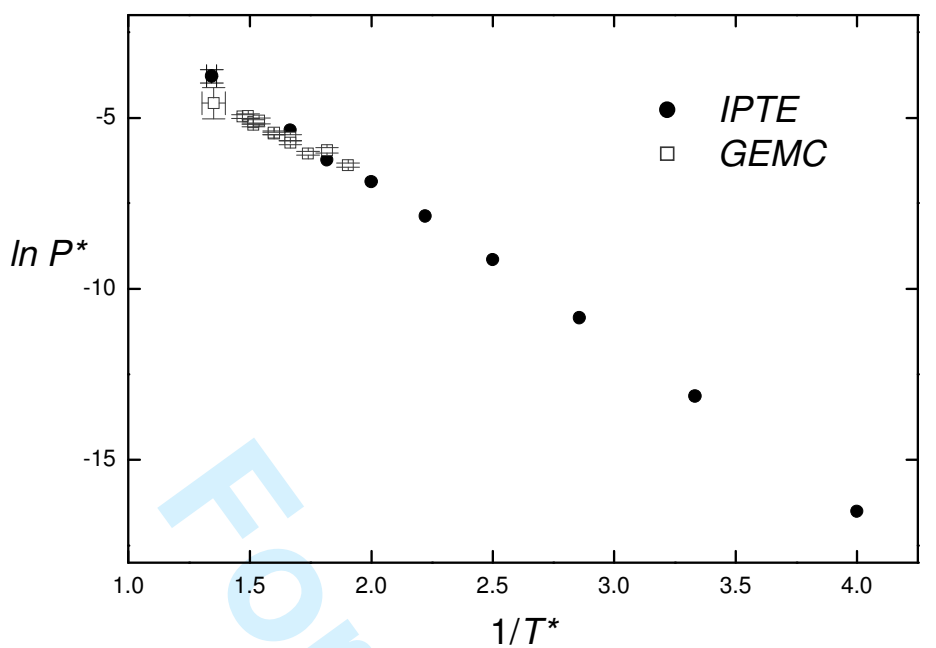

Figure 6.

(a)

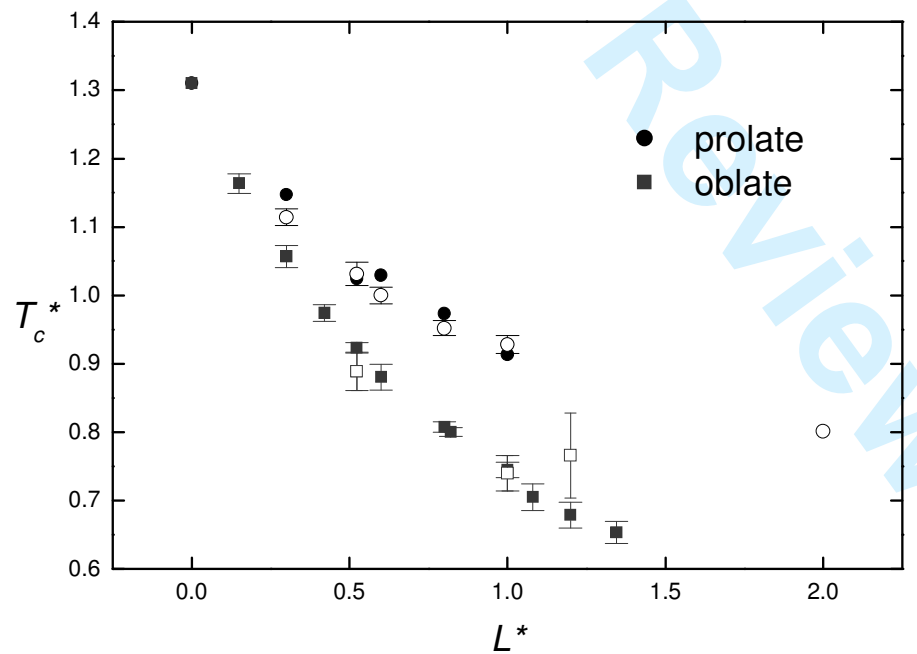

(b) 


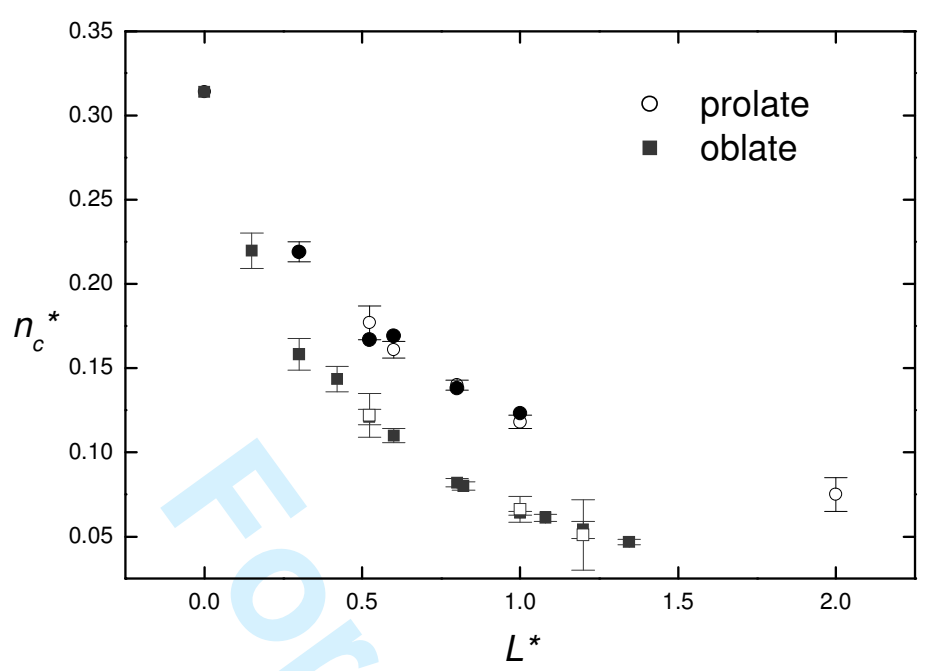

(c)

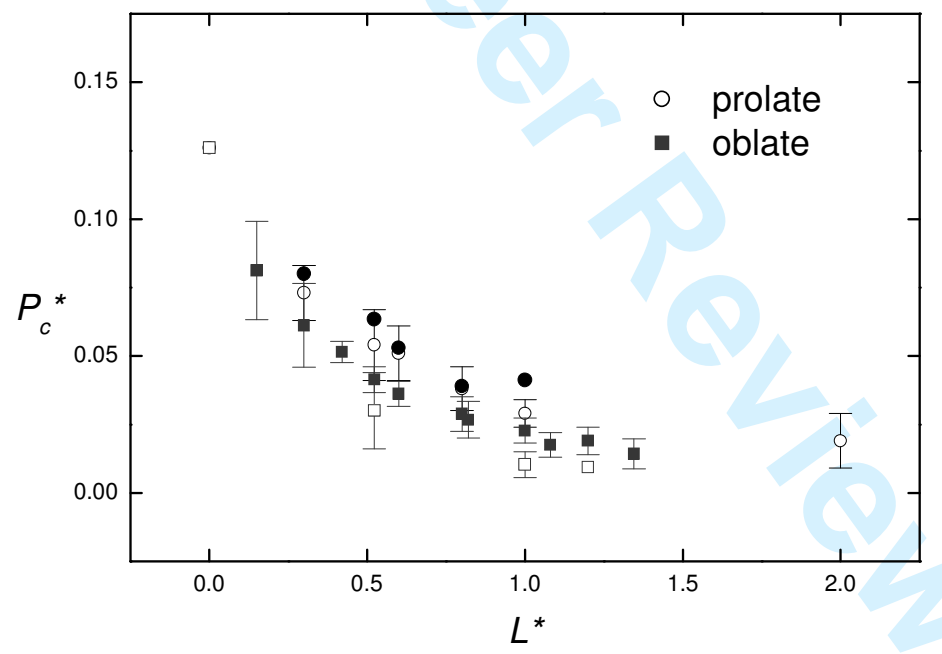

Figure 7.

(a) 


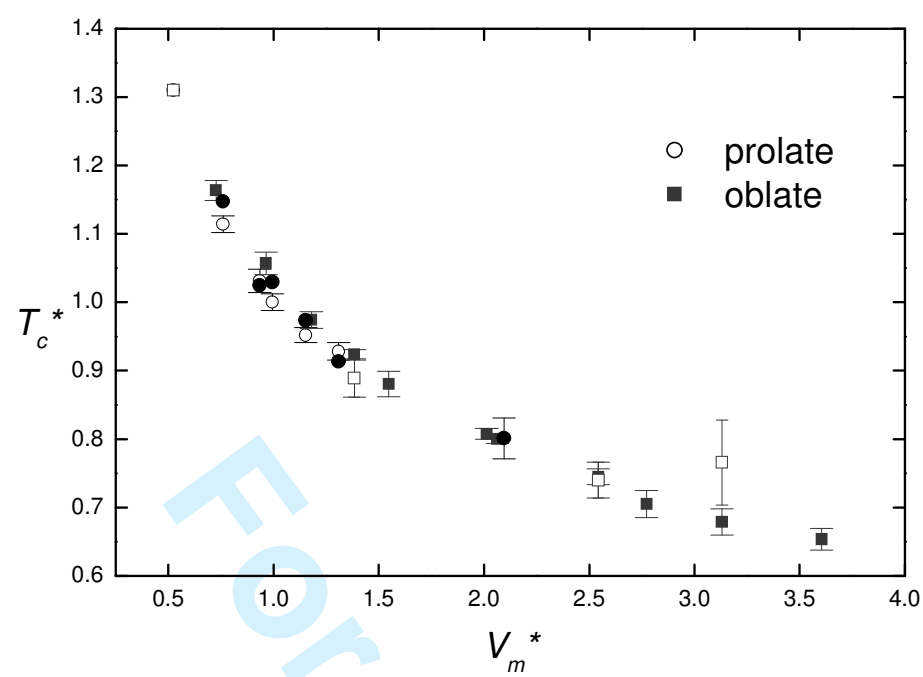

(b)

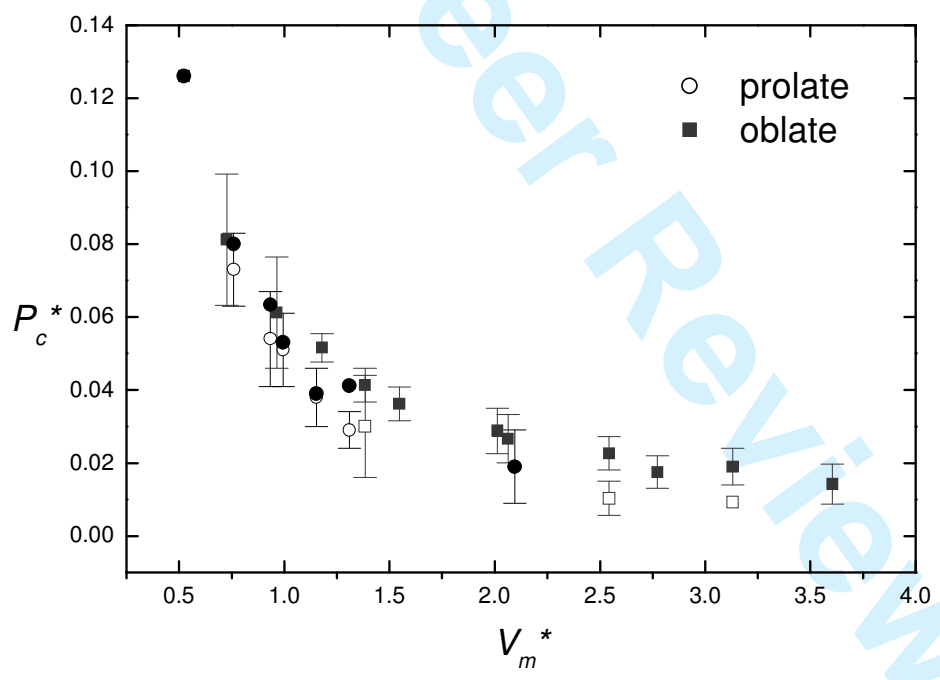

(c) 


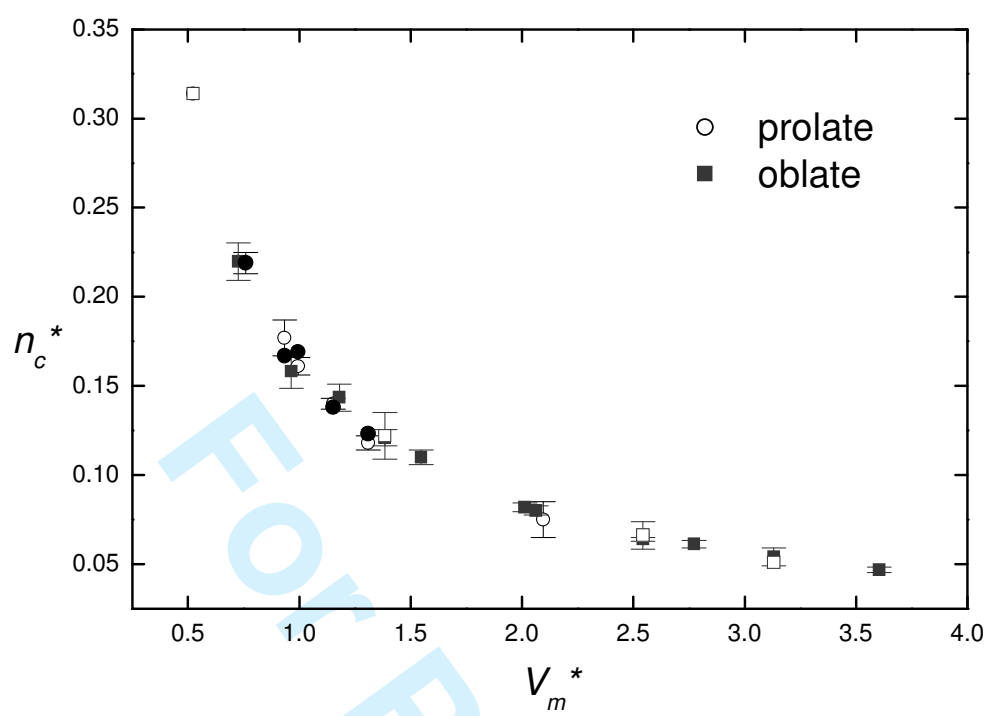

Figure 8.

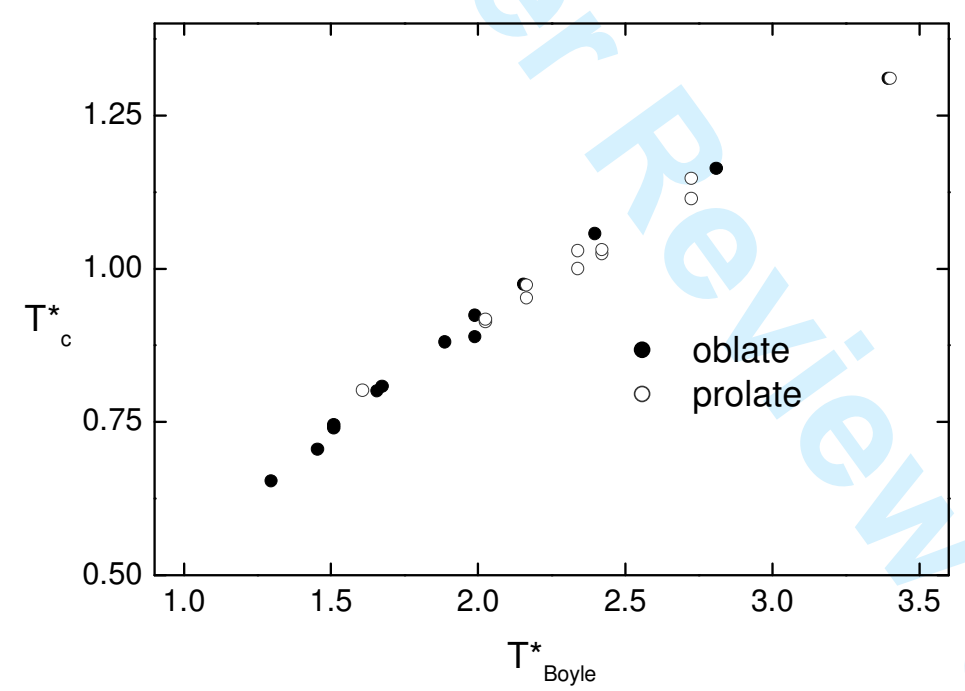


$7 / 12 / 201001: 54$

\title{
Vapour-liquid equilibrium of fluids composed by oblate
}

\section{molecules}

\author{
Francisco Gámez ${ }^{1}$, Santiago Lago ${ }^{1, *}$, Benito Garzón ${ }^{2}$, Patrick Merkling ${ }^{1}$ and Carlos \\ $\operatorname{Vega}^{3}$ \\ ${ }^{1}$ Dpto. Sistemas Físicos, Químicos y Naturales. \\ Fac. Ciencias Experimentales, \\ Ctra.Utrera Km.1, \\ Univ. Pablo de Olavide, \\ 41013 Sevilla, Spain \\ ${ }^{2}$ Departamento de Física Aplicada, Fisicoquímica y Óptica, \\ Facultad de Farmacia, \\ Universidad San Pablo CEU,
}

Urb. Monteprincipe Boadilla del Monte 28660,

Spain

${ }^{3}$ Dpto. Química Física

Fac. CC. Químicas,

Univ. Complutense

28040 Madrid, Spain

* Author to whom correspondence should be addressed: slagara@upo.es 
ABSTRACT

Gibbs ensemble Monte Carlo simulations are performed to obtain the vapourliquid equilibrium of oblate-like fluids interacting through the Kihara intermolecular potential. Our results confirm the validity of a perturbation theory for Kihara fluids, whose accuracy for prolate fluids was tested some years ago. As in the case of hard ellipsoids, the symmetry of the phase diagram of oblate and prolate models is analyzed. An interesting relation of Boyle temperature and critical parameters with molecular volume is found for the considered models. As a particular application, this relation allows the prediction of some thermodynamic properties of a new promising biofuel 2,5dimethylfuran.

\section{Keywords}

Vapour-liquid equilibrium, oblate molecules, Boyle temperature, intermolecular potential. 


\section{1.- INTRODUCTION}

The liquid state is the most difficult to study among the classical states of matter. Powerful methods to predict thermodynamic and transport properties of liquids have been proposed during the second half of the past century but most of these methods are reliable only for simple fluids [1]. However, most liquids are formed by polyatomic molecules where in addition to translational degrees of freedom, one must also consider rotation and the internal vibrations of the molecule [2]. The inclusion of rotational degrees of freedom, even in rigid models (where vibrations are not considered), requires some additional information about the mass distribution in the molecule or the molecular shape. Classical rotation movement is described using the inertia tensor which can be written as a $3 \times 3$ square matrix. This matrix can be diagonalized and the three eigenvalues define the so-called principal moments of inertia (PMIs) and the eigenvectors define the principal axes of the molecule with origin at the centre of mass of the molecule. Molecules with low symmetry have three different PMIs and are called asymmetric tops [2]. The decay time of several properties of asymmetric tops depends on three diffusion coefficients and five relaxation times [3]. However, experiments can determine only three coefficients or times. In this case, it is very usual to consider that two of the three moments are equal and the third one is a revolution axis for the molecule [3]. This is equivalent to approximate the asymmetric top as a symmetric top [2]. If the principal moment of inertia with respect to the revolution axis is the largest we have an oblate molecule, if it is the smallest, the molecule is called prolate. Whether a molecule is prolate or oblate can be inferred from the experiment. Many real-world molecules can be considered prolate or oblate. Liquids composed by prolate molecules have received a lot of theoretical attention and thermodynamic properties have been 
qualitatively and quantitatively reported for molecules of this geometry. For prolate molecules a number of studies describing liquid crystal transitions [4], vapour-liquid equilibrium [5] and glassy behaviour [6] have been reported. However, relatively little effort has been directed to obtaining properties of oblate-like fluids [7-9]. In some cases, oblate molecules are not described by pure oblate models but rather by related models [10-13]. A new phenomenology appears in oblate molecules, such as discotic liquid crystal phases with short axes parallel to the director, formation of fragile glasses like in o-terphenyl, peculiar surface properties like in asphaltenes [14], ... Oblate-like molecules are candidates for specific molecular architectures, and some of them mainly aromatic carboxylic acids such as 3-hydroxypicolinic or gentisic acid- have absorption, stability and solubility properties suitable for MALDI matrices [14]. In biology, red blood cells are the most common cells with oblate shape. Important gasliquid interchange takes place in the quaternary structure of hemoproteins contained in these cells, where hemo groups embed gas molecules in a reversible way [15]. Even a prolate-oblate transition has been observed in natural systems such as bilayer membranes [16]. Moreover, an oblate molecule, 2,5-dimethylfuran, has been recently proposed as biofuel [17] and we dedicate some of our work to this molecule. From a chemical point of view, it is well-known that the $\pi$-electronic cloud in common oblate systems is often delocalized, yielding chemical reactivity which is so different from that of prolate molecules that they are considered as different functional groups, so called aromatics. Swimming in the very diverse behaviour of oblate-like molecules, our goals in this paper are to answer the following unsolved questions: i) Does molecular shape affect the vapour-liquid equilibrium?; ii) Is it possible to find a symmetry in the phase diagram of oblate and prolate Kihara spherocylinders as found in hard ellipsoids?[18]. In order to solve these questions, this paper is scheduled as follows: section 2 is devoted 
to present the model and the relevant parameters in prolate and oblate systems. In section 3 we present the simulation procedure and a review of the perturbation theory used in this work. Results related to vapour-liquid equilibrium of some oblate molecules including the new biofuel 2,5-dimethylfuran are shown in section 4. Finally, some remarks and conclusions close the paper in section 5 . 


\section{Molecular model and hard convex systems.}

The Kihara potential describes the interaction between two molecules of any shape as:

$$
u_{i j}^{K}=4 \varepsilon\left[\left(\frac{\sigma}{\rho_{i j}}\right)^{12}-\left(\frac{\sigma}{\rho_{i j}}\right)^{6}\right]
$$

where $\sigma$ and $\varepsilon$ are length and energy parameters and $\rho$ is the shortest distance between molecular hard cores, depending not only on centre-to-centre distance but on mutual molecular orientations. As usual, the core of a spherical molecule is considered to be a dot, for a prolate molecule the core is considered to be a segment and for an oblate molecule the core is an infinitely thin disk, as considered in convex geometry. For more clarity see figure 1. Hard convex bodies and thermodynamic properties have been widely studied and many equations of state have been established [19]. All these equations of state relate thermodynamic quantities with three geometrical descriptors: $(1 / 4 \pi)$ times the mean intrinsic curvature $(\mathcal{R})$, surface $(S)$ and volume $(\mathcal{V})$. Moreover, an analytical equation relates the second virial coefficient of hard convex bodies, $B_{2}$, to the non sphericity parameter $\alpha=\frac{R S}{3 \mathcal{V}}$ in the form:

$$
\frac{B_{2}}{\mathcal{V}}=1+3 \alpha
$$

This equation is a particular case of the general equation: 


$$
B_{2}(T)=\frac{1}{2}\left[V_{c+c}-\int f(\rho) S_{c+\rho+c} d \rho\right]
$$

with $S_{c+\rho+c}=2 S_{c}+8 \pi R_{c} R_{c}+8 \pi\left(R_{c}+R_{c}\right) \rho+4 \pi \rho$

where $f(\rho)=\exp (-\beta u)-1$ is the Mayer function and the geometrical quantities $V_{c+c}$ and $S_{c+\rho+c}$ depends on surface, volume and $(1 / 4 \pi)$ times the mean curvature of the hard convex nucleus. As it has been mentioned, we have considered a hard rod as the core of the prolate model and an infinitely thin disk as the core of the oblate one, whose characteristic parameters are well known to be different. For a given $L^{*}=L / \sigma$ (prolate, see fig.1) or $D^{*}=D / \sigma$ (oblate, see fig. 1 ), the second virial coefficient will be different due to the difference in the geometrical properties (surface, volume and mean radius of curvature of the hard convex core). For prolate molecules the geometrical properties of the core have been given elsewhere $[11,13]$. For oblate molecules they are given by

$$
\begin{aligned}
& R_{c}=\frac{\sigma}{2}\left(1+\frac{\pi D^{*}}{4}\right) \\
& S_{c}=\frac{\pi \sigma^{2}}{2}\left(D^{* 2}+\pi D^{*}+2\right) \\
& V_{c}=\frac{\pi \sigma^{3}}{24}\left(6 D^{* 2}+3 \pi D^{*}+4\right)
\end{aligned}
$$

Hereinafter, we shall use $L^{*}$ to denote the anisotropy of both prolate and oblate molecules (although it should be clear that in the case of oblate molecules $L^{*}$ is just $\left.D^{*}\right)$. In figure 2, the Boyle temperature (where the second virial coefficient $\mathrm{B}_{2}$ becomes zero) is plotted as a function of $L^{*}$. It is obvious that for a given value of $L^{*}$ the prolate and oblate Kihara models differ in their Boyle temperatures. However, it is possible to map a prolate model into an oblate model by imposing the condition of equal Boyle temperature, that is: 


$$
T_{B}^{*}\left(L_{1}^{*}\right)=T_{B}^{*}\left(D_{2}^{*}\right)
$$

where $L^{*}$ on the left hand side represents the anisotropy of a prolate model and $D^{*}$ on the right hand side represents the anisotropy of the oblate model. In figure 3, the Boyle temperature of prolate and oblate molecules is plotted as a function of the molecular volume. The very remarkable result is that oblate Kihara molecules and prolate Kihara molecules present the same Boyle temperature when they have the same reduced volume. Thus Eq. (7) is satisfied (approximately) when both the prolate and oblate molecules present the same reduced volume. A new and important question arises now: will the symmetry between prolate and oblate Kihara molecules exhibited at the Boyle temperature be also present in the rest of the phase diagram? This is one of the questions we intend to address here. In the case of hard ellipsoids a symmetry in the phase diagram was found [20] based on the fact that a hard prolate ellipsoid with aspect ratio $\kappa$ has the same volume than an oblate one with aspect ratio $1 / \kappa$. In this work, we shall determine the vapour liquid equilibria for prolate and oblate molecules by computer simulation to explore the existence of symmetry between prolate and oblate models (when described by the Kihara model). It will be shown that when the comparison is made between models having the same molecular volume there is a good mapping between prolate and oblate models 


\section{Computer simulation and perturbation theory.}

\section{3a. Computer simulations.}

In previous works, we have widely established vapour-liquid equilibria of prolate-like Kihara molecules [5], even including multipolar interactions [21,22]. For that purpose, an algorithm able to calculate several millions of $\rho$ per second is used [23]. In this work a well tested algorithm to calculate the minimum distance between disks has been employed [8]. In analogy to the case of prolate molecules, Gibbs ensemble Monte Carlo (GEMC) simulations [24] are developed for systems with three different reduced diameters $L^{*}=L / \sigma$ equal to $0.5233,1$ and 1.2 , in order to compare with prolate models with the same $L^{*}$ value. GEMC were performed with 512 particles, 6000-10000 cycles for equilibration and $6000-8000$ for averages, truncating the potential when $\rho_{\mathrm{c}}=3 \sigma$ and adding long tail corrections according to expression given by:

$$
\begin{aligned}
& p^{L R}=-\frac{2}{3} n^{2} \pi \int_{\rho_{c}}^{\infty} r_{12}^{3}\left\langle\frac{\partial u\left(r_{12}, \Omega_{1}, \Omega_{2}\right)}{\partial r_{12}}\right\rangle_{g} d r_{12} \\
& U^{L R}=2 n \pi \int_{\rho_{c}}^{\infty} r_{12}^{2}\left\langle u\left(r_{12}, \Omega_{1}, \Omega_{2}\right)\right\rangle_{g} d r_{12}
\end{aligned}
$$

where the subscript $g$ means geometrical average. Furthermore, long range corrections can be obtained analytically [25] by rewriting the previous equations using convex body geometry. 


\section{3b. Perturbation theory.}

A few years ago, we have presented an accurate perturbation theory for Kihara prolate molecules. This theory is a second order perturbation theory based on MoGubbins division [26] of the Kihara potential:

$$
\begin{aligned}
& u_{0}\left(r_{12}, \Omega_{1}, \Omega_{2}\right)= \begin{cases}u^{K}\left(r_{12}, \Omega_{1}, \Omega_{2}\right)+\varepsilon & \rho \leq \sigma \sqrt[6]{2} \\
0 & \rho>\sigma \sqrt[6]{2}\end{cases} \\
& u_{1}\left(r_{12}, \Omega_{1}, \Omega_{2}\right)=\left\{\begin{array}{cc}
-\varepsilon & \rho \leq \sigma \sqrt[6]{2} \\
u^{K}\left(r_{12}, \Omega_{1}, \Omega_{2}\right) & \rho>\sigma \sqrt[6]{2}
\end{array}\right.
\end{aligned}
$$

It allows us to write the Helmholtz free energy up to second order in perturbation expansion as:

$$
\frac{A^{\text {res }}}{N k T}=\frac{A_{0}^{\text {res }}}{N k T}+\frac{A_{1}}{N k T}+\frac{A_{2}}{N k T}
$$

Since the residual free energy is considered to be the one associated to a hard particle with the same radius and diameter, the contribution to Helmholtz free energy is measured by means of a BLIP expansion and integration of the Boublík's equation of state for hard convex body [27], which gives:

$$
\frac{A_{0}^{r e s}}{N k T}=\frac{\eta\left[6 \alpha^{2}-2 \alpha+\eta\left(\frac{3}{2} \alpha(3-5 \alpha)\right)\right]}{(1-\eta)^{2}}+\left(6 \alpha^{2}-5 \alpha-1\right) \ln (1-\eta)
$$

where $\eta=n / V$ is the packing fraction. Perturbation terms can be calculated in different ways: 
1).- The first order perturbation term is calculated by solving the OrnsteinZernike equation with Percus-Yevick (PY), Hypernetted-chain (HNC) or Reference Hypernetted-chain (RHNC) closure with the background correlation function $y\left(r, \Omega_{1}, \Omega_{2}\right)=g\left(r, \Omega_{1}, \Omega_{2}\right) \exp \left[\beta u\left(r, \Omega_{1}, \Omega_{2}\right)\right]$ extracted from Reference Average Mayer function potential (RAM):

$$
\Phi^{R A M}(r)=-\varepsilon T^{*} \ln \int\left\langle u_{0}\left(\mathbf{r}_{\mathbf{1 2}}, \Omega_{1}, \Omega_{2}\right)\right\rangle_{g} d \Omega_{1} d \Omega_{2}
$$

and finally:

$$
\frac{A_{1}}{N k T}=\frac{2 n \pi}{k T} \int_{0}^{\infty} r_{12}^{2}\left\langle u_{1}\left(r_{12}, \Omega_{1}, \Omega_{2}\right) \exp \left(-\beta u_{0}\left(r_{12}, \Omega_{1}, \Omega_{2}\right)\right)\right\rangle_{g} y^{R A M}(r) d \mathbf{r}
$$

In a previous paper, it was shown that similar results for soft repulsive bodies obtained either using the non-sphericity parameter $\alpha$ extracted from the geometrical relationship and the background correlation function from RHNC equation, or using $\alpha$ from virial coefficient (see equation 2) and background correlation function from PY approximation. Due to the faster convergence of the PY formalism, the second choice is used because results obtained with both integral equations are very close.

2).- Two different choices for the second contribution term can be used [28]: local compressibility approximation (LCA) or macroscopic compressibility approximation (MCA): 


$$
\begin{aligned}
& \frac{A_{2}^{L C A}}{N k T}=-\frac{n \pi}{k T}\left(\frac{\partial n}{\partial p}\right) \int_{0}^{\infty} r_{12}^{2}\left\langle u_{1}^{2}\left(r_{12}, \Omega_{1}, \Omega_{2}\right) \exp \left(-\beta u_{0}\left(r_{12}, \Omega_{1}, \Omega_{2}\right)\right)\right\rangle_{g} y^{R A M}(r) d \mathbf{r} \\
& \frac{A_{2}^{M C A}}{N k T}=-\frac{n \pi}{k T}\left(\frac{\partial n}{\partial p}\right)_{0} \frac{\partial}{\partial p}\left(n \int_{0}^{\infty} r_{12}^{2}\left\langle u_{1}^{2}\left(r_{12}, \Omega_{1}, \Omega_{2}\right) \exp \left(-\beta u_{0}\left(r_{12}, \Omega_{1}, \Omega_{2}\right)\right)\right\rangle_{g} y^{R A M}(r) d \mathbf{r}\right)
\end{aligned}
$$

As for the first order term, the convenience of using MCA approximation has been previously tested and will be used in this work.

Once the Helmholtz free energy is determined, one calculates the equation of state and internal energy through:

$$
\begin{aligned}
& Z=1+n^{*}\left(\frac{\partial\left(A^{r e s} / N k T\right)}{\partial n^{*}}\right)_{T} \\
& \frac{U^{r e s}}{N k}=\left(\frac{\partial\left(A^{r e s} / N k T\right)}{\partial(1 / T)}\right)_{n}
\end{aligned}
$$

and the vapour-liquid equilibrium is solved treating vapour phase as a virial expansion up to second order and solving the nonlinear system:

$$
\begin{aligned}
& n_{l} Z_{l}=n_{g}\left(1+n_{g} B_{2}\right) \\
& \frac{A_{l}}{N k T}+Z_{l}+\ln n_{l}=n_{g} B_{2}+\left(1+n_{g} B_{2}\right)+\ln n_{g}
\end{aligned}
$$


As it is well known, analytical perturbation theories cannot predict critical properties, and numerical extrapolation methods are commonly necessary. To overcome this problem, an extrapolation method for the theory already described (IPT) [29] was derived based on deviations between Monte Carlo and theoretical calculations of the first order contribution to Helmholtz free energy, giving very accurate predictions of VLE for prolate Kihara molecules yielding the so-called IPTE.

\section{RESULTS}

In figures 4 and 5, vapour-liquid coexistence of oblate and prolate Kihara molecules respectively for $L^{*}\left(D^{*}\right)=1.0$ are shown. Numerical results for the VLE of simulated models are shown in table 1 for oblate models and in table 2 for prolate models not previously reported. The critical temperature is accurately predicted for both shapes (prolate or oblate) using IPTE as can be observed in table 3. However, liquid density is systematically underestimated in the case of oblate molecules while the agreement for prolate molecules is excellent. Better agreement is obtained in considering coexistence pressures. In panel $b$ of figures 4 and 5, Clausius plots for these models are presented. IPTE data fit very well the simulation results. Furthermore, although densities in the VLE curve are not accurately estimated for oblate models with high anisotropy, critical densities are. As pointed out above, the behaviour of Boyle temperature against molecular volume is universal, but not against characteristic length. In figure 6 , the behaviour of critical parameters against $L^{*}$ is shown, while in figure 7 the plot against molecular volume can be observed. Surprisingly, critical parameters also exhibit universal behaviour against molecular volume, within the simulation error in the case of GEMC results, independently of shape. Table 3 summarizes all the results 
considered here by simulation and perturbation theory. A comparison for both models can be observed plotting critical temperature against Boyle temperature. Thus, knowledge of Boyle temperature could be used as a tool to estimate critical temperature. A plot of critical temperature against Boyle temperature is presented in figure 8 showing the universal character of the relation. This rule can be used when molecular volume is not too high. This plot allows to predict critical properties of important but scarcely studied molecules such as the biofuel 2,5-dimethyl furan [17] whose known thermodynamic properties are practically restricted to its boiling point. In this case, we have checked using a quantum chemical package (gaussian03 at the B3LYP/6-31G**) that the molecule is perfectly planar and the dipole moment is negligible (0.16 Debye). Using van der Waals radii for atoms on this geometry-optimized structure yields $D^{*}=1.6$ and $V_{\mathrm{m}}{ }^{*}=2.83$. From the fit of $T_{\mathrm{c}}{ }^{*}$ vs. $V_{\mathrm{m}}{ }^{*}$ (figure $7 \mathrm{a}$ ), one can interpolate to obtain $T_{\mathrm{c}}{ }^{*}=0.57$. Moreover, using the classical empirical approximation [31] of $\mathrm{T}_{\text {boiling }} / \mathrm{T}_{\text {critical }}=2 / 3$ and the experimental value for boiling temperature of $346 \mathrm{~K}$, one can predict for this biofuel $T_{\mathrm{c}}=550 \mathrm{~K}, T_{\mathrm{B}}=1117 \mathrm{~K}$. Moreover, from the relation between $T_{\mathrm{c}}{ }^{*}$ and $T_{\mathrm{c}}$ an estimated value of $\varepsilon / k=966 \mathrm{~K}$ is found. This value is consistent with the usual values adopted for benzene or cyclohexane in this potential $[9,11]$.

\section{CONCLUSIONS.}

Gibbs ensemble Monte Carlo simulations of oblate and prolate Kihara molecules have been carried out in order to estimate the influence of shape upon vapour-liquid equilibrium. This influence is very subtle and, thus, we have found a similar behaviour of the virial coefficient -i.e. equation of state- for both models when it is plotted against molecular volume. The same behaviour is observed when critical parameters are plotted 
against molecular volume which opens the possibility of predicting molecular properties of poorly studied fluids.

\section{ACKNOWLEDGMENTS}

This work was supported by grants BFM2003-07055, FIS2007-66079-C02-01, and CTQ2007 60910 of the Spanish Ministerio de Educación y Ciencia. 


\section{BIBLIOGRAPHY}

[1] J.P.Hansen and I.R.MacDonald, Theory of Simple Liquids, $3^{\text {rd }}$.ed., Academic Press, 2006

[2] D.A.McQuarrie and J.D.Simon, Physical chemistry. A molecular approach, chapter 18, university Science Books, Sausalito,1997

[3] J.R.Lakowicz, Principles of Fluorescence Spectroscopy, $2^{\text {nd }}$. ed., chapter 12, Kluwer, New York,1999

[4] A. Cuetos, B. Martinez-Haya, L.F. Rull and S. Lago, J.Chem.Phys. 117, 2934-2946, (2002); (E) J.Chem.Phys. 117,11405,(2002).

[5] C.Vega, S.Lago, E.de Miguel and L.F.Rull, J.Phys.Chem.96,7431-7437, (1992).

[6] S.Lago, M.Cortada, M.D.Lobato, F.Gámez, M.Basoalto J.M.Pedrosa and S.Calero, J.Mol.Liq.134,136-141,(2007).

[7] M. Wojcik, K. E. Gubbins, Molec. Phys. 53 (2): 397-420, (1984).

[8] P. Kadlec, J. Janecek, T. Boublik, Molec. Phys. 98 (8): 473-479, (2000).

[9] J. Janecek, T. Boublik, Molec. Phys. 98(11),765-767, (2000).

[10] B. M. Mulder, Molec. Phys. 103 (10): 1411-1424, (2005).

[11] S.Lago and T.Boublík,Coll.Czech.Chem.Commun.,45,3051-3056,(1980).

[12] C.Vega, S.Lago and P.Padilla, J.Phys.Chem.96,1900-1905,(1992).

[13] S.Lago, J.L.López-Martín, B.Garzón y C.Vega, J.Phys.Chem.98,53555361,(1994).

[14] M.D.Lobato, J.M.Pedrosa, A.R.Hortal,B.Martínez-Haya, R.Lebrón-Aguilar and S.Lago, Colloid Surf.A298,72-79,(2007).

[15] A. E. Hoetink, T. J. C. Faes, K. R. Visser, R. M. Heethaar, IEEE Trans. Biomedical Engn. 51 (7): 1251-1261, (2004). 
[16] M. Jaric, U. Seifert, W. Wintz, M. Wortiz. Phys. Rev E., 52, 6623-6634, (1995).

[17] L.D.Schmidt and P.J.Dauenhauer, Nature, 447,914,(2007).

[18] D. Frenkel and B. M. Mulder, Mol. Phys. 55 (5): 1171-1192, (1985).

[19] T.Boublík, Collection Czechoslovak Chem. Commun, vol 51,2301-2432, (1986).

[20] D. Frenkel, B. M. Mulder,J. P. McTague, Phys. Rev. Lett. 52 (4): 287-290, (1984).

[21] B.Garzón,S.Lago, C.Vega and L.F.Rull, J.Chem.Phys.102, 7204-7216, (1995).

[22] B.Garzón, S.Lago, C.Vega, E.de Miguel and L.F.Rull, J.Chem.Phys.101, 4166-4176, (1994).

[23] C.Vega and S.Lago, Comput.Chem.18,55-59, (1994).

[24].-A.Z.Panagiotopoulos, Molec. Phys. 61, 813-826, 1987

[25] C.Vega and D.Frenkel, Molec.Phys., 67,633-650, (1989).

[26] K. C. Mo and K. E. Gubbins. Chem. Phys. Lett. 27: 144-148. (1974).

[27] T.Boublík, Molec. Phys., 42, 209, (1981).

[28] J. A. Barker, D. Henderson. Rev. Mod. Phys. 48 (4): 587-671 1976

[29] C.Vega and S.Lago, Chem.Phys.Lett.185,516-521, (1991).

[30] A. Lotfi, J. Vrabec and J. Fischer, Mol. Phys. 76(6) 1319 (1992).

[31]G.Z.Guldberg, Z.Phys.Chem. 5, 374, (1890). A more accessible reference is R.Digilov and M.Reiner, Eur.J. Phys. 25,15-22 (2004) 
TABLES

Table 1. GEMC coexistence data for oblate fluids.

\begin{tabular}{cccc}
\multicolumn{3}{c}{$D^{*}=0.5233$} \\
\hline$T^{*}$ & $n^{*}$ gas & $n^{*}$ liq & $p^{*}$ \\
\hline 0.79 & $0.024_{4}$ & $0.23_{2}$ & $0.0143_{5}$ \\
0.78 & $0.0255_{2}$ & $0.25_{1}$ & $0.0144_{4}$ \\
0.775 & $0.0125_{4}$ & $0.243_{4}$ & $0.0083_{4}$ \\
0.775 & $0.0149_{8}$ & $0.228_{8}$ & $0.0095_{5}$ \\
0.77 & $0.0155_{5}$ & $0.242_{8}$ & $0.0098_{3}$ \\
0.75 & $0.0165_{3}$ & $0.26_{1}$ & $0.0099_{3}$ \\
0.725 & $0.0142_{8}$ & $0.273_{5}$ & $0.0084_{4}$ \\
0.7 & $0.0110_{6}$ & $0.281_{5}$ & $0.0065_{3}$ \\
0.675 & $0.0088_{4}$ & $0.290_{4}$ & $0.0051_{2}$ \\
0.65 & $0.0056_{2}$ & $0.296_{3}$ & $0.002_{2}$ \\
0.625 & $0.0047_{7}$ & $0.306_{4}$ & $0.002_{2}$ \\
\hline
\end{tabular}

$D^{*}=1$

\begin{tabular}{cccc}
\hline$T^{*}$ & $n^{*}{ }_{\text {gas }}$ & $n^{*}$ liq & $p^{*}$ \\
\hline 0.68 & $0.0141_{9}$ & $0.11_{1}$ & $0.0070_{4}$ \\
0.67 & $0.0145_{2}$ & $0.13_{1}$ & $0.0071_{6}$ \\
0.66 & $0.0104_{5}$ & $0.13_{1}$ & $0.0055_{3}$ \\
0.66 & $0.0118_{7}$ & $0.128_{9}$ & $0.0059_{4}$ \\
0.65 & $0.0131_{5}$ & $0.140_{5}$ & $0.0062_{5}$ \\
0.625 & $0.0084_{3}$ & $0.146_{3}$ & $0.0043_{2}$ \\
0.625 & $0.0087_{3}$ & $0.146_{4}$ & $0.00434_{25}$ \\
0.6 & $0.0080_{5}$ & $0.1556_{25}$ & $0.0038_{3}$ \\
0.6 & $0.0065_{2}$ & $0.1565_{25}$ & $0.0032_{2}$ \\
0.575 & $0.0047_{2}$ & $0.161_{2}$ & $0.00238_{13}$ \\
0.55 & $0.00583_{9}$ & $0.169_{2}$ & $0.0026_{2}$ \\
0.525 & $0.0038_{2}$ & $0.173_{1}$ & $0.0017_{1}$ \\
\hline
\end{tabular}

\begin{tabular}{cccc}
\multicolumn{4}{c}{$D^{*}=1.2$} \\
\hline$T^{*}$ & $n^{*}{ }_{\text {gas }}$ & $n^{*}$ liq & $p^{*}$ \\
\hline 0.425 & $0.00793_{1}$ & $0.1523_{5}$ & $0.0014_{6}$ \\
0.475 & $0.00872_{3}$ & $0.1495_{6}$ & $0.0016_{7}$ \\
0.5 & $0.00999_{6}$ & $0.1445_{8}$ & $0.0027_{6}$ \\
0.525 & $0.0106_{1}$ & $0.1393_{15}$ & $0.0033_{5}$ \\
0.55 & $0.0107_{1}$ & $0.1359_{14}$ & $0.0037_{4}$ \\
0.575 & $0.0112_{2}$ & $0.1284_{25}$ & $0.00431_{4}$ \\
0.6 & $0.01002_{15}$ & $0.120_{2}$ & $0.0043_{4}$ \\
\hline
\end{tabular}


Table 2. GEMC coexistence data for prolate fluids.

a) $L^{*}=0.5233$. Critical parameters are: $T_{\mathrm{c}}^{*}=1.03(2), n_{\mathrm{c}}{ }^{*}=0.18(1)$ and $p_{\mathrm{c}}{ }^{*}=0.054(12)$.

b) $L^{*}=1$. Critical parameters are: $T_{\mathrm{c}}{ }^{*}=0.928(13), n_{\mathrm{c}}{ }^{*}=0.118(4)$ and $p_{\mathrm{c}}{ }^{*}=0.029(5)$.

c) $L^{*}=1.2$. Critical parameters are: $T_{\mathrm{c}}{ }^{*}=0.911(13), n_{\mathrm{c}}{ }^{*}=0.106(5)$ and $p_{\mathrm{c}}{ }^{*}=0.030(14)$.

$L^{*}=0.5233$

\begin{tabular}{cccc}
\hline$T^{*}$ & $n^{*}$ gas & $n^{*}$ liq & $p^{*}$ \\
\hline 0.95 & $0.0520_{23}$ & $0.31_{2}$ & $0.0328_{15}$ \\
0.925 & $0.043_{2}$ & $0.337_{14}$ & $0.0280_{12}$ \\
0.9 & $0.039_{2}$ & $0.36_{1}$ & $0.025_{1}$ \\
0.875 & $0.0286_{15}$ & $0.364_{13}$ & $0.0194_{9}$ \\
0.85 & $0.024_{2}$ & $0.380_{7}$ & $0.016_{1}$ \\
0.8 & $0.017_{1}$ & $0.399_{6}$ & $0.0112_{7}$ \\
0.775 & $0.012_{1}$ & $0.407_{4}$ & $0.0084_{7}$ \\
0.75 & $0.0094_{6}$ & $0.418_{4}$ & $0.00634_{35}$ \\
\hline
\end{tabular}

$$
L^{*}=1
$$

\begin{tabular}{cccc}
\hline$T^{*}$ & $n^{*}$ gas & $n^{*}{ }_{\text {liq }}$ & $p^{*}$ \\
\hline 0.825 & $0.024_{1}$ & $0.23_{2}$ & $0.0151_{6}$ \\
0.8 & $0.020_{1}$ & $0.246_{9}$ & $0.0123_{6}$ \\
0.775 & $0.0150_{7}$ & $0.261_{7}$ & $0.0095_{4}$ \\
0.75 & $0.0126_{4}$ & $0.272_{7}$ & $0.0079_{2}$ \\
0.725 & $0.010_{1}$ & $0.281_{6}$ & $0.0063_{5}$ \\
0.7 & $0.0083_{4}$ & $0.289_{3}$ & $0.0051_{2}$ \\
0.675 & $0.0058_{3}$ & $0.297_{4}$ & $0.0036_{2}$ \\
0.65 & $0.0051_{3}$ & $0.306_{3}$ & $0.0030_{2}$ \\
0.625 & $0.00343_{23}$ & $0.312_{4}$ & $0.0020_{1}$ \\
0.6 & $0.0028_{5}$ & $0.3194_{25}$ & $0.0016_{2}$ \\
\hline
\end{tabular}

\begin{tabular}{cccc}
\multicolumn{4}{c}{$L^{*}=1.2$} \\
\hline$T^{*}$ & $n^{*}{ }_{\text {gas }}$ & $n^{*}$ liq $_{1}$ & $p^{*}$ \\
\hline 0.7 & $0.0095_{17}$ & $0.249_{3}$ & $0.0055_{8}$ \\
0.675 & $0.0074_{4}$ & $0.259_{4}$ & $0.0043_{2}$ \\
0.65 & $0.0055_{4}$ & $0.265_{4}$ & $0.0032_{2}$ \\
0.625 & $0.00435_{24}$ & $0.275_{3}$ & $0.0025_{1}$ \\
0.6 & $0.0029_{2}$ & $0.2794_{35}$ & $0.0016_{1}$ \\
0.575 & $0.00195_{3}$ & $0.287_{3}$ & $0.00107_{2}$ \\
0.55 & $0.00160_{5}$ & $0.291_{3}$ & $0.00084_{3}$ \\
0.5 & $0.0014_{1}$ & $0.3050_{25}$ & $0.00066_{6}$ \\
0.475 & $0.00079_{4}$ & $0.3100_{25}$ & $0.00037_{2}$ \\
0.7 & $0.010_{2}$ & $0.2496_{34}$ & $0.0055_{8}$ \\
\hline
\end{tabular}


Table 3. Critical properties of oblate fluids.

\begin{tabular}{|c|c|c|c|c|c|c|c|}
\hline$D^{*}$ & $T_{\mathrm{c}}^{*}($ IPTE $)$ & $T_{\mathrm{c}} *(\mathrm{GEMC})$ & $n_{\mathrm{c}}{ }^{*}($ IPTE $)$ & $n_{\mathrm{c}} *(\mathrm{GEMC})$ & $p_{\mathrm{c}}{ }^{*}($ IPTE$)$ & $p_{\mathrm{c}}^{*}(\mathrm{GEMC})$ & $Z_{c}$ \\
\hline 0 & & $1.310^{\mathrm{a}}$ & & $0.314^{\mathrm{a}}$ & & $0.126^{\mathrm{a}}$ & \\
\hline 0.3 & $1.06_{2}$ & & $0.169_{9}$ & & $0.061_{15}$ & & 0.342 \\
\hline 0.42 & $0.974_{12}$ & & $0.143_{5}$ & & $0.052_{3}$ & & 0.373 \\
\hline 0.5233 & $0.923_{7}$ & $0.89_{3}$ & $0.121_{4}$ & $0.122_{13}$ & $0.041_{4}$ & $0.030_{14}$ & 0.367 \\
\hline 0.6 & $0.88_{2}$ & & $0.110_{4}$ & & $0.036_{4}$ & & 0.351 \\
\hline 0.8 & $0.808_{7}$ & & $0.082_{2}$ & & $0.029_{6}$ & & 0.438 \\
\hline 0.82 & $0.800_{6}$ & & $0.080_{2}$ & & $0.027_{6}$ & & 0.422 \\
\hline 1 & $0.745_{11}$ & $0.74_{3}$ & $0.064_{1}$ & $0.066_{8}$ & $0.023_{4}$ & $0.010_{5}$ & 0.482 \\
\hline 1.08 & $0.70_{2}$ & & $0.061_{2}$ & & $0.018_{4}$ & & 0.418 \\
\hline 1.2 & $0.68_{2}$ & $0.77_{6}$ & $0.054_{5}$ & $0.05_{2}$ & $0.019_{5}$ & $0.009_{3}$ & 0.518 \\
\hline 1.345 & $0.653_{15}$ & & $0.047_{1}$ & & $0.014_{5}$ & & 0.456 \\
\hline
\end{tabular}

${ }^{a}$ data taken from ref. 30 


\section{FIGURE CAPTIONS}

Figure 1. Molecular cores of a) spherical molecules; b) linear (prolate) molecules and c) oblate molecules.

Figure 2. Boyle temperature against elongation (prolate model) or diameter (oblate model).

Figure 3. Boyle temperature against molecular volume of prolate and oblate models obtained by IPTE.

Figure 4. VLE properties of prolate Kihara fluids with $L^{*}=1.0$ calculated by IPTE (solid symbols) and GEMC (solid symbols). a) Coexistence densities. b) vapour pressures.

Figure 5. VLE properties of oblate Kihara fluids with $D^{*}=1.0$ calculated. a) Coexistence densities. b) vapour pressures. Symbols as in figure 4

Figure 6. Critical properties of several prolate and oblate fluids with different sizes. a) Temperatures. b) Densities. c) Pressures. Solid symbols as in figure 4.

Figure 7. Dependence of critical parameters on molecular volume for oblate and prolate Kihara fluids.

Figure 8. Critical versus Boyle temperatures for oblate and prolate Kihara models as obtained by IPTE. 


\section{FIGURES}

\section{Figure 1.}

a)

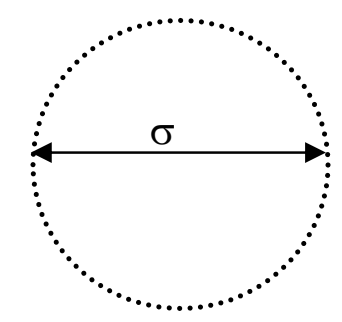

b)

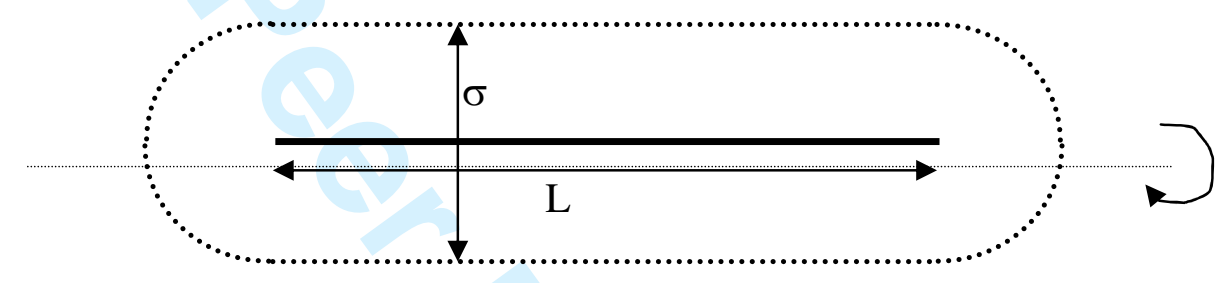

c)

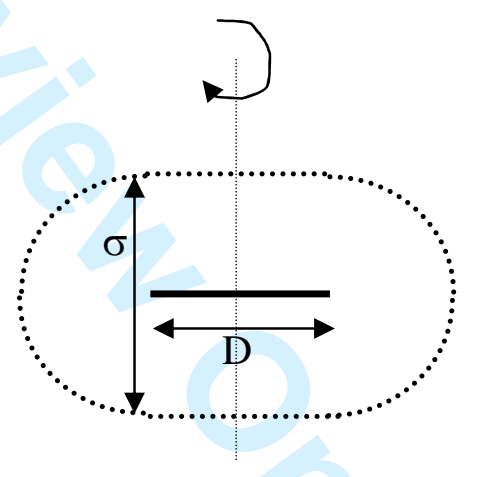

Figure 2. 


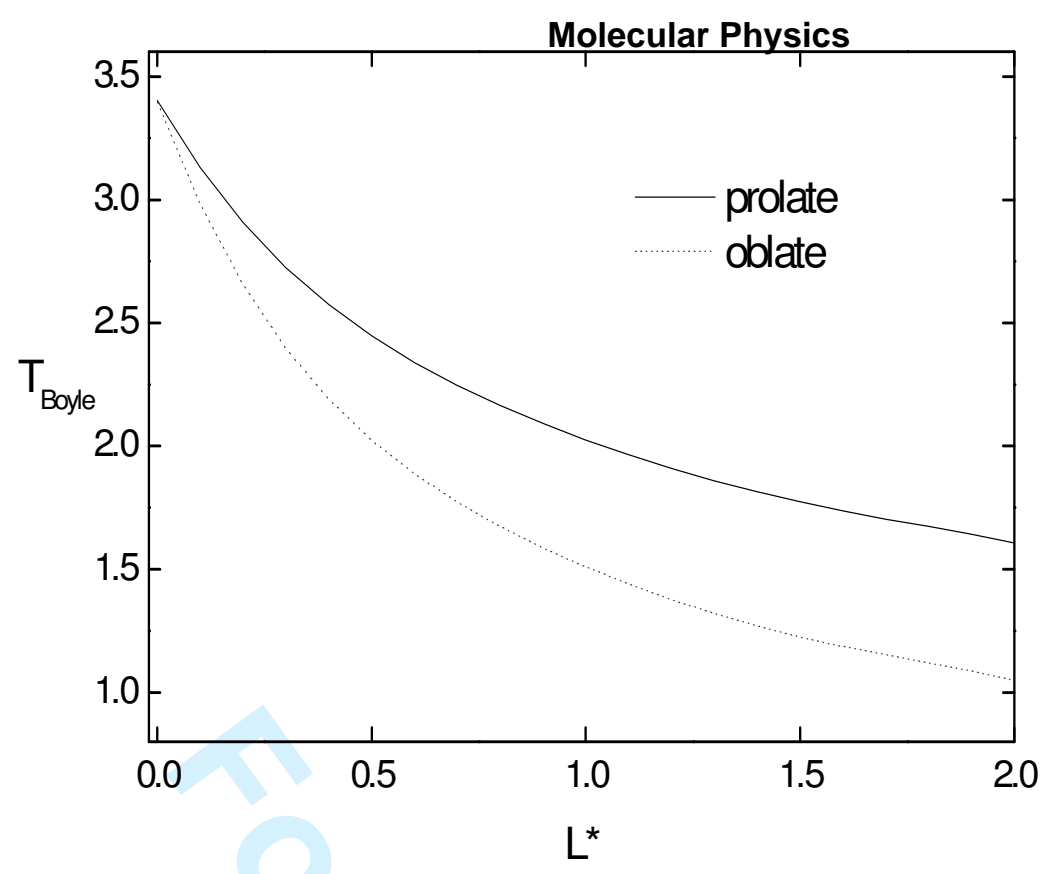

Page 52 of 58 
Figure 3.

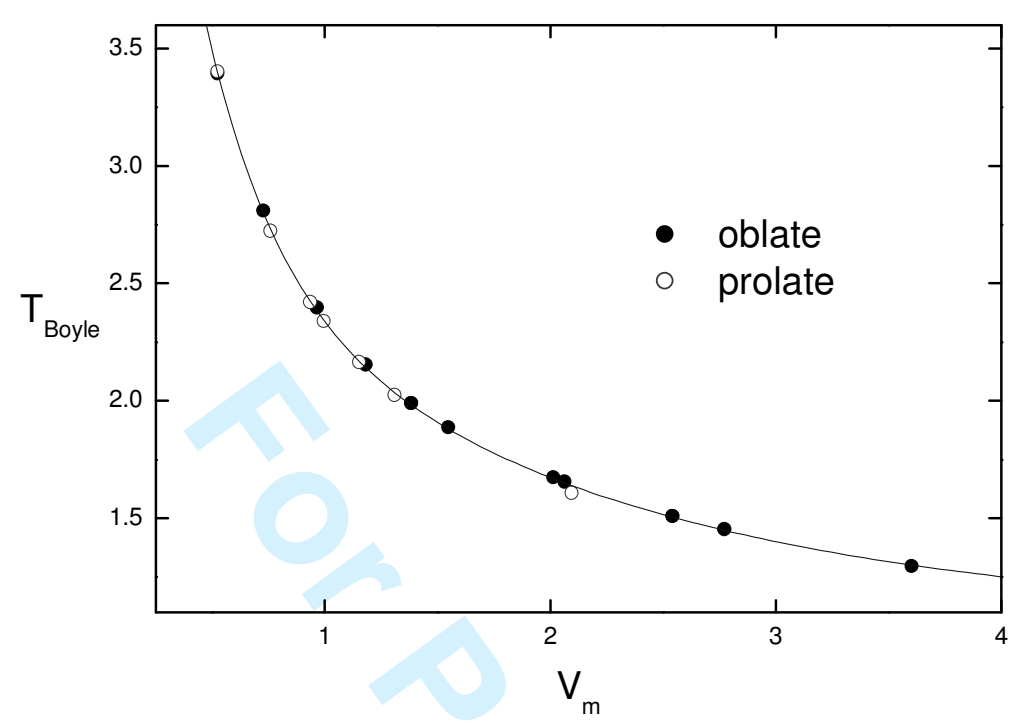

Figure 4.

(a)

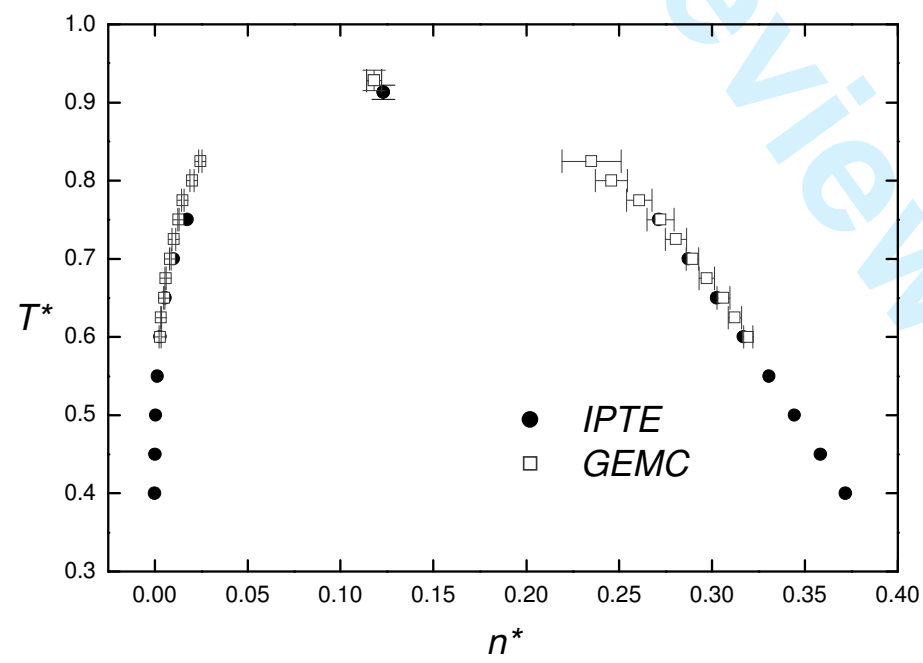




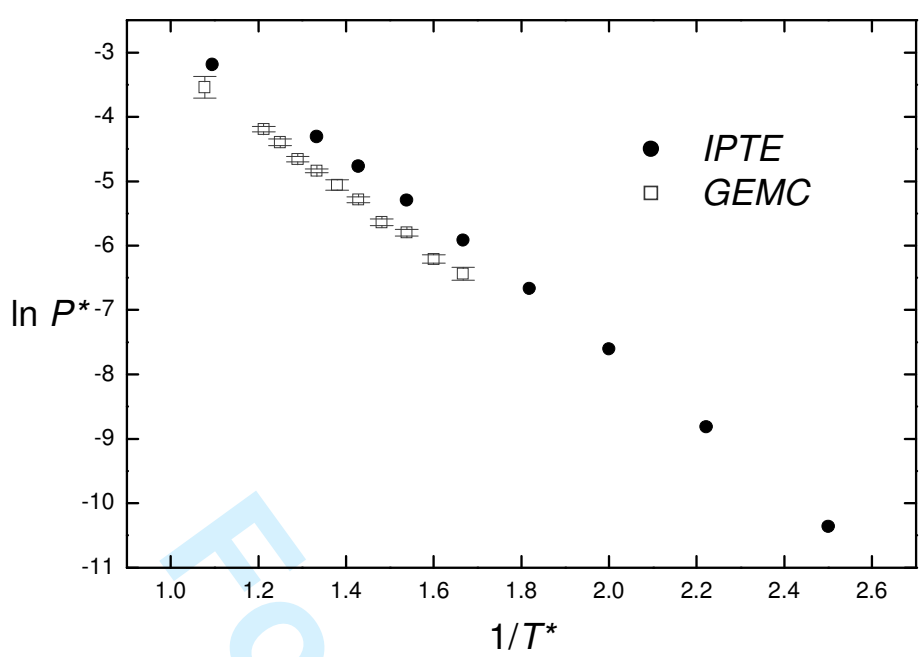

Figure 5.

(a)

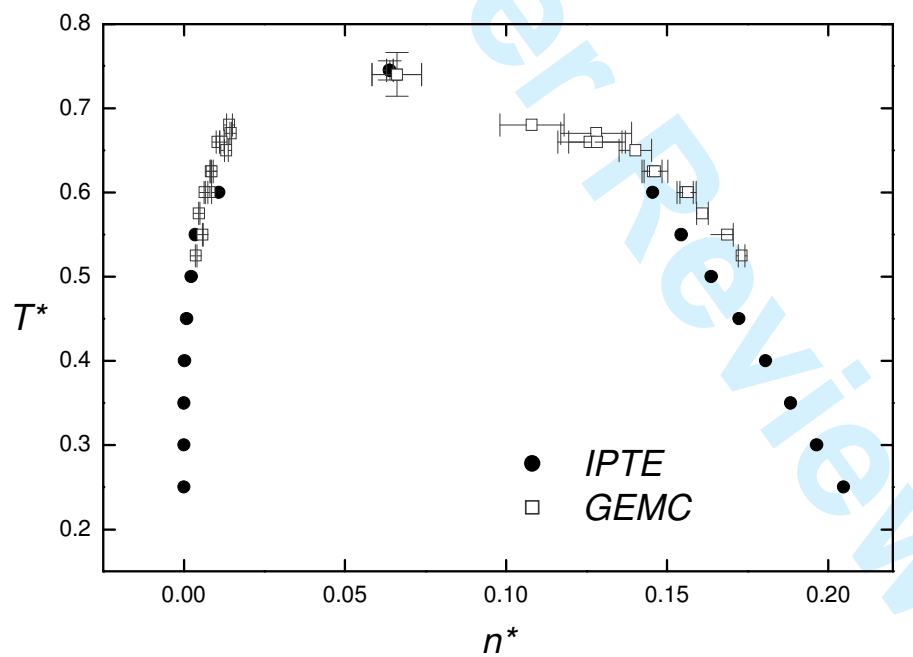

(b) 


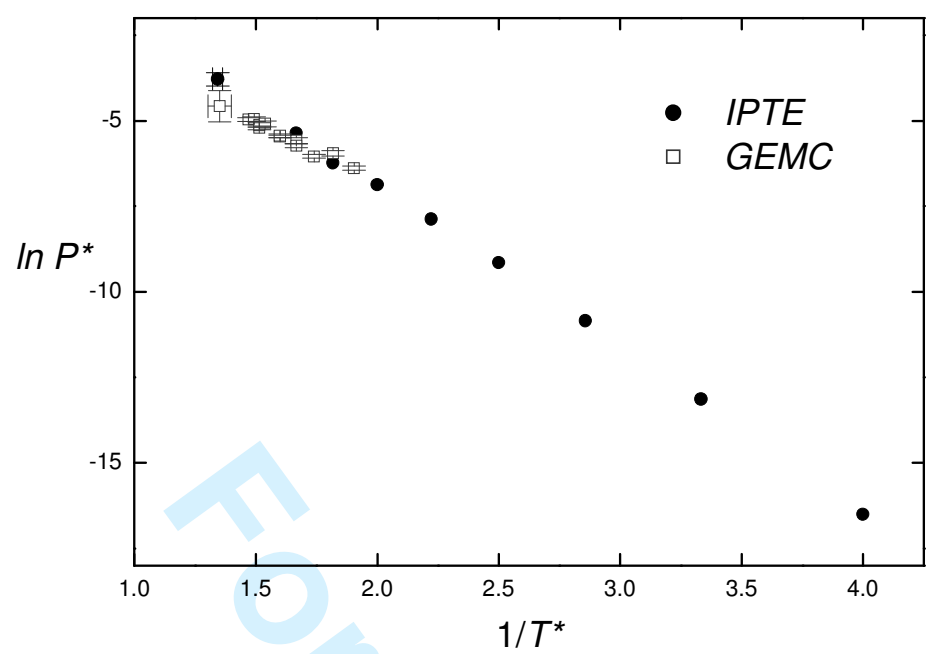

Figure 6.

(a)

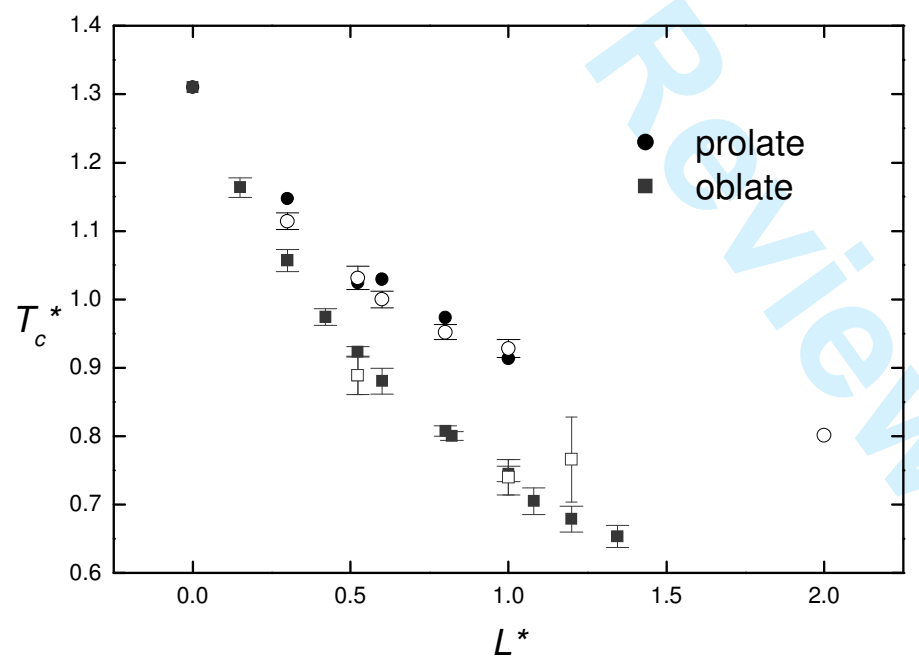

(b) 


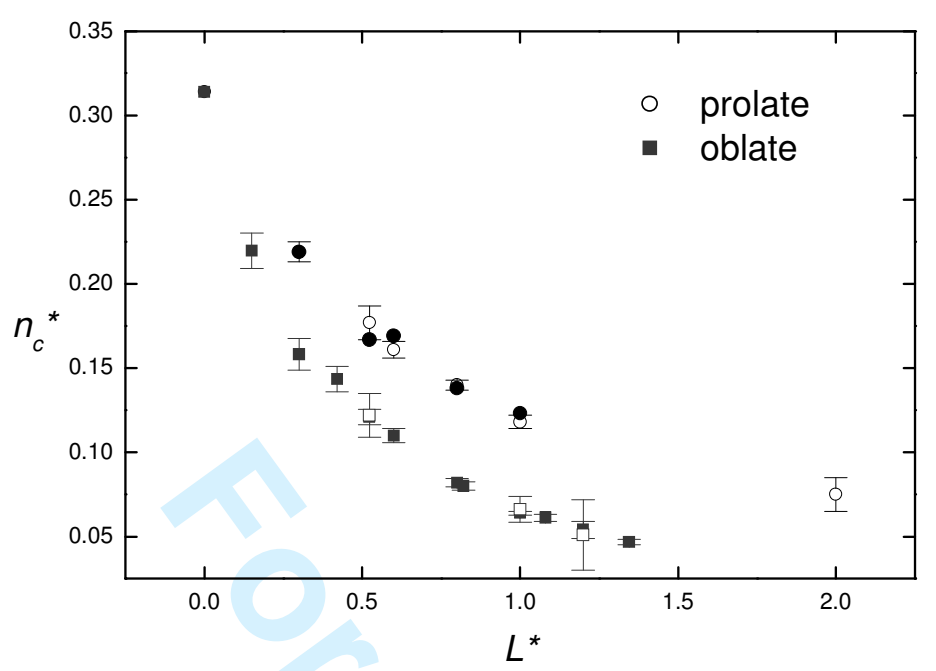

(c)

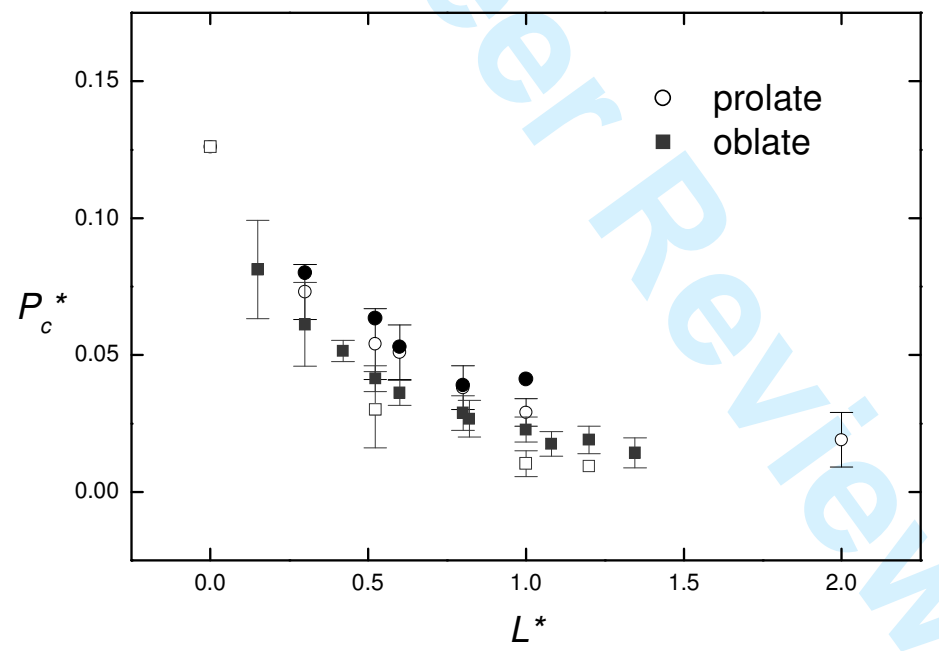

Figure 7.

(a) 


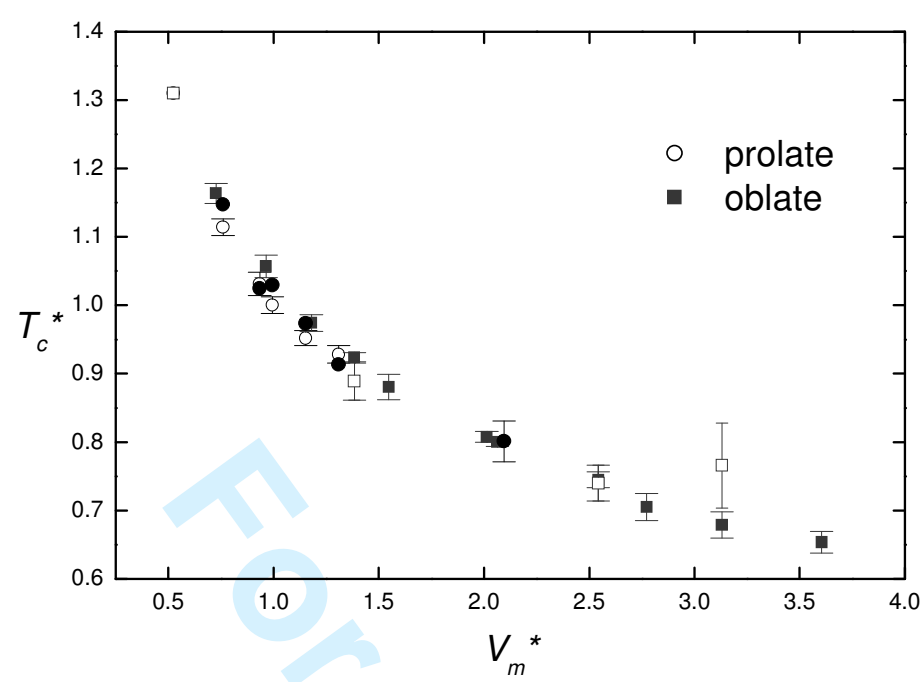

(b)

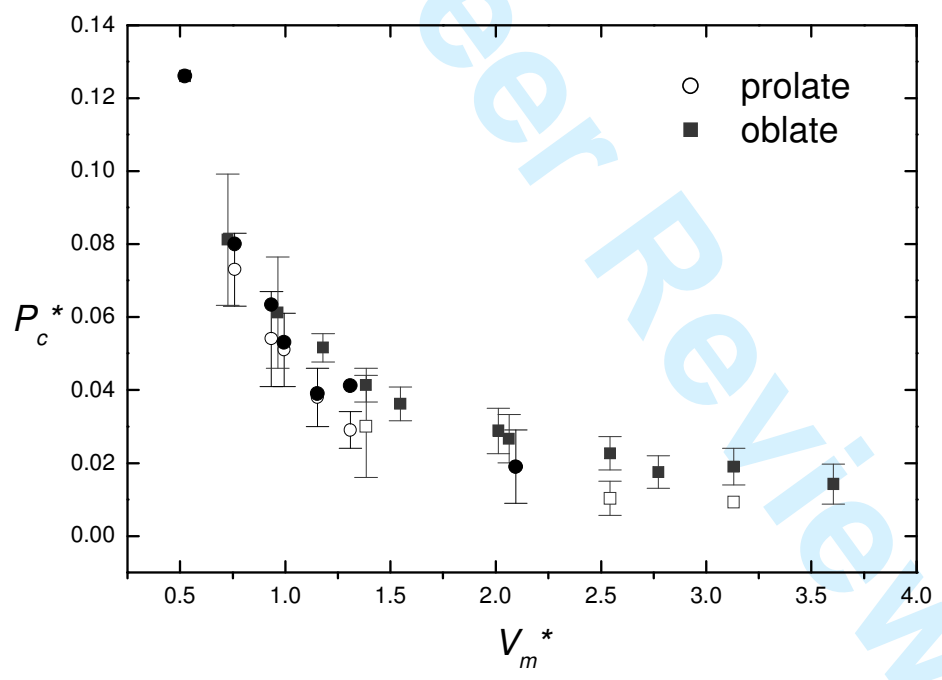

(c) 


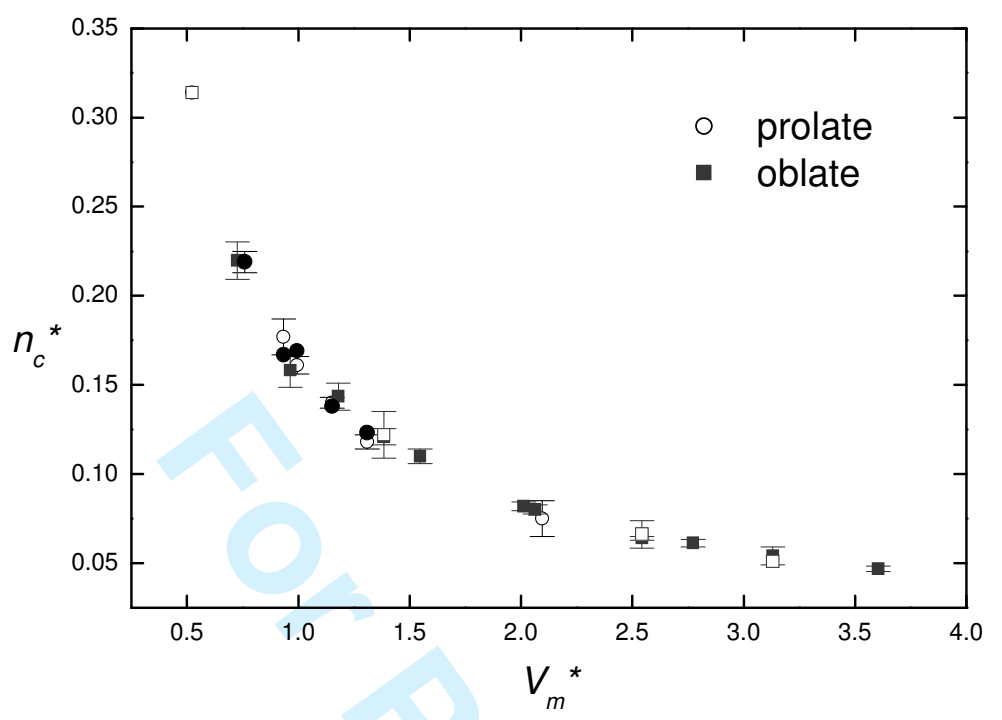

Figure 8.

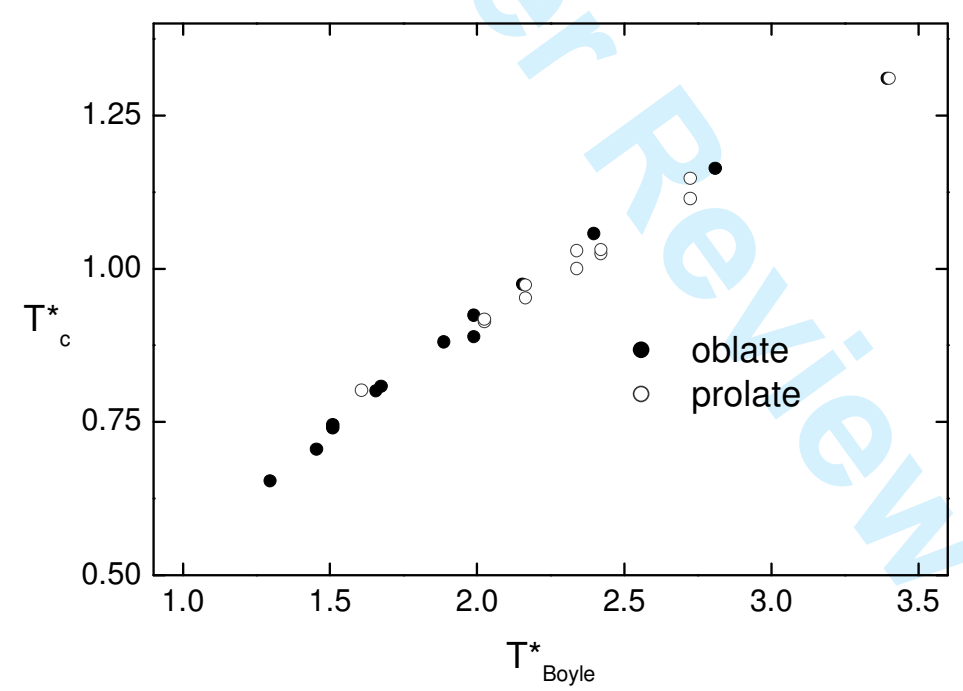

URL: http://mc.manuscriptcentral.com/tandf/tmph 